\title{
SUPRAMOLECULAR ASSEMBLY OF HUMAN PULMONARY SURFACTANT PROTEIN SP-D
}

R. Arroyo ${ }^{1}$, A. Martin-González ${ }^{2}$, M. Echaide' A. Jain $^{3}$, W. H. Brondyk ${ }^{3}$, J. Rosenbaum $^{4}$, F. Moreno-Herrero' ${ }^{2}$,J. Perez-Gil ${ }^{1}$

${ }^{1}$ Department of Biochemistry, Faculty of Biology, Complutense University, Madrid, Spain

2Department of Macromolecular Structures, National Center of Biotechnology, CSIC, Madrid, Spain

${ }^{3}$ Sanofi, Framingham, MA, U.S.A

${ }^{4}$ Research \& Development Dept., Airway Therapeutics LLC., Cincinnati OH, U.S.A.

Corresponding Author: Jesús Pérez-Gil

Dept. Biochemistry, Fac. of Biology

Complutense University

Jose Antonio Novais 12

28040 Madrid, Spain

Key words:

Lung surfactant, SP-D, collectins, AFM, non-covalent interactions 


\section{GRAPHICAL ABSTRACT}

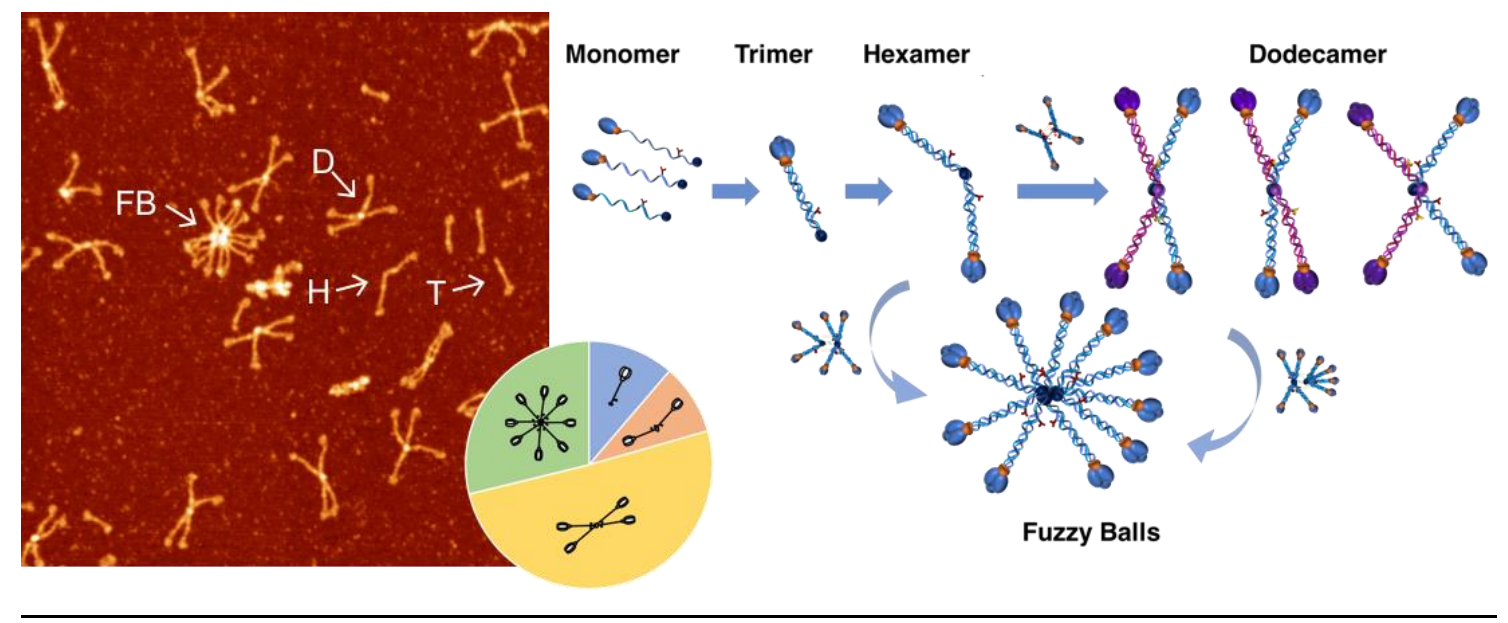




\section{HIGHLIGHTS}

- $\quad$ rhSP-D and hSP-D are found as trimers, hexamers, dodecamers and larger oligomers.

- Dodecamers are the most abundant oligomer of the protein.

- Hexamers play an important role in the oligomerization pathway of SP-D.

- Non-covalent interactions are important to form and maintain dodecamers and larger oligomers.

- Distribution and 3D confomartion of SP-D oligomers is sensitive to environmental conditions such as $\mathrm{pH}$. 


\begin{abstract}
$\underline{\text { ABSTRACT }}$
Pulmonary surfactant protein D (SP-D) is a glycoprotein from the collectin family that takes part in the lung surfactant system. It exhibits host defense and immune regulatory functions within the lung. It is known that SP-D monomer forms trimers, which associate into higher order oligomers. However, the pathway and the interactions involved in the assembly of SP-D oligomers are not clearly understood. In the current study, a recombinant form of human SP-D (rhSP-D) has been studied by Atomic Force Microscopy (AFM) and electrophoresis, with the goal of analyzing, qualitative and quantitatively, the conformational diversity and the determinants defining the oligomerization pathway of the protein. The studied rhSP-D is a mixture of trimers, hexamers, dodecamers and higher order oligomeric species, with dodecamers accounting for more than $50 \%$ of the protein by mass. Similar structures were also found in hSP-D obtained from proteinosis patients. The proportion of dodecamer is increased under acidic conditions, accompanied by a conformational change into more compact configurations. Dodecamers were found to be necessarily formed by two hexamers joined through non-covalent ionic and hydrophobic interactions between the N-terminal domains and the nearby area of SP-D collagen stems.
\end{abstract}

\title{
ABBREVIATIONS
}

SP-A: surfactant protein A

SP-D: surfactant protein D

CRD: carbohydrate recognition domain

CHO: Chinese hamster ovary

DHFR: dihydrofolate reductase

PAP: pulmonary alveolar proteinosis

GA: glutaraldehyde 


\section{INTRODUCTION}

Lung collectin proteins, Surfactant Protein A (SP-A) and Surfactant Protein D (SP-D), are glycoproteins of the calcium-dependent lectin family (C-type lectins) [1-3], found in lung surfactant and in extra-pulmonary mucosal tissues [1, 4].

SP-D was first described as CP4 protein by Persson and colleagues in 1988 [5]. They characterized a $43 \mathrm{kDa}$ product from type II pneumocytes, composed of collagenous and non-collagenous domains and the presence of asparagine-Nlinked carbohydrates $[5,6]$. Numerous structural and functional studies have been carried out since then to further characterize SP-D from several organisms. Moreover, different recombinant versions of SP-D have been produced, with Chinese hamster ovary (CHO) cells being the most widely-used system to produce the human recombinant protein (rhSP-D) [7,8].

SP-D is involved in the innate immune defense system of the lung, where it participates in pathogen clearance by two actions: i) it directly agglutinates and eliminates bacteria through the mucociliary transport of upper airways and ii) it mediates the removal of pathogens through phagocytosis by macrophages [4]. Moreover, SP-D participates in the metabolism and clearance of lung surfactant [18].

SP-D monomer contains 355 amino acids $(43 \mathrm{kDa})$ arranged in four structural domains (see supplementary figure $\mathrm{S} 1$ ), including: i) an N-terminus domain, where two conserved cysteines (C15 and C20) are important for protein oligomerization [9], ii) a collagen domain, composed by a sequence of Gly-X-Y triplets, iii) an $\alpha$-helical coiledcoil neck region and, iv) a globular domain, the carbohydrate recognition domain (CRD). The CRD contains four additional cysteine residues implicated in intramolecular disulphide bonds [10], and four calcium-binding sites important for its calcium-dependent immune activity [11-13]. The sequence of SP-D also contains an asparagine in position 70, which is glycosylated, and a number of lysines that are partially O-glycosylated [14]. Most prolines, typically in position Yaa, the third residue relative to glycine in collagen domain triplets, are hydroxylated. Three monomers form trimers through the folding of the collagenous region into triple helices and the assembly of a coiled-coil bundle of $\alpha$-helices in the neck region. It has been described that trimers associate into higher order oligomers such as dodecamers, which are structures formed by four trimers, or the so called "fuzzy balls", larger oligomers composed by 6 or more trimers, existing in a wide and variable range of sizes. Whether the different oligomeric forms of SP-D might have selective 
contributions to SP-D-related activities is unknown, as well as how such oligomerization-function determinants could be modulated by physiologically relevant factors. This gap in knowledge is in part due to the difficulties of characterizing in detail, both in qualitative and quantitative terms, the conformational diversity of SP-D under different environmental constraints. New tools are also needed to dissect the protein domains or regions, and the nature of the interactions, involved in the formation and stabilization of the different protein oligomers.

In the present study, we have analyzed in detail the structure of a recombinant human SP-D produced in CHO cells, with the goal of assessing, qualitative and quantitatively, the conformational diversity and the determinants defining the oligomerization pathway of the protein. Using novel atomic force microscopy (AFM) and electrophoresis methodologies, we have described the different SP-D oligomeric forms, and defined and quantitatively characterized some of their relevant features. The distribution of oligomeric forms of rhSP-D has been for first time quantified as a function of $\mathrm{pH}$. In addition, we have validated that the oligomeric forms found in rhSP-D are also present in a natural hSP-D preparation. Our results indicate that rhSP-D and hSP-D are assembled in four oligomeric states: trimers, hexamers, dodecamers and larger aggregates (fuzzy balls), with dodecamers being the most abundant structure in all experimental conditions tested. We have developed a crosslinking protocol to detect the presence of SP-D dodecameric forms by PAGE-SDS, in which dodecamer is only visualized after chemical crosslinking and in the presence of denaturing agents, indicating the importance of ionic and hydrophobic interactions in dodecamer formation and stabilization. 


\section{MATERIALS AND METHODS}

\section{Recombinant human SP-D (rhSP-D)}

A dihydrofolate reductase (DHFR)-deficient CHO cell line that was adapted to animal derived component-free media was transfected with an expression vector containing human SP-D cDNA (Met-11, Thr-160, Ser-260). Transcription of the human SP-D cDNA was driven by the hamster $\beta$-actin enhancer-promoter region and the termination and polyadenylation was regulated by the SV40 polyadenylation signal. Also on the expression vector is a DHFR expression cassette. Generation of stably transfected $\mathrm{CHO}$ pools was completed by selecting the transfected cells with methotrexate. Identification of $\mathrm{CHO}$ clones expressing a high level of recombinant SP-D was completed by a flow cytometric method described previously [19]. In this method, the human CD52 sequence is placed upstream of the SP-D cDNA and this CD52 utilizes a non-AUG initiation codon, which results in inefficient translation of CD52 compared to the translation of AUG-containing SP-D. The expression level of the CD52 reporter is correlated with the expression of SP-D and high expressing SPD clones were sorted by flow cytometry using a labeled anti-CD52 antibody. SP-D expressing $\mathrm{CHO}$ cells were grown in $\mathrm{CD} \mathrm{CHO}$ media (Thermo Fisher) to generate recombinant SP-D. The purification of rhSP-D was completed with a capture step modified from the previously described method, followed by a proprietary carbohydrate affinity step [7]. In the modified method, the anion exchange resin used for the capture step was ceramic $Q$ hyperD (Pall Corp, 20066-015). The resin was first equilibrated with $25 \mathrm{mM}$ Tris, $0.01 \%$ Tween 80, $\mathrm{pH}$ 7.4, and then conditioned medium diluted 1:1 with $25 \mathrm{mM}$ Tris, $\mathrm{pH} 7.4$ was loaded onto the resin, followed by a wash step with $25 \mathrm{mM}$ Tris, $100 \mathrm{mM} \mathrm{NaCl}, \mathrm{pH} 7.4$ and the rhSP-D was eluted with $25 \mathrm{mM}$ Tris, 1.2 M NaCl, pH 7.4. All resins were sanitized with $0.2 \mathrm{M} \mathrm{NaOH}$. The endotoxin level of the material used in this study was $<1 \mathrm{EU} / \mathrm{mg}$. The purified SP-D was buffer exchanged into $20 \mathrm{mM}$ Tris, $1 \mathrm{mM}$ EDTA, $200 \mathrm{mM} \mathrm{NaCl}, \mathrm{pH} 7.4$ and stored at $-80{ }^{\circ} \mathrm{C}$.

More than 10 different SP-D clones have been analysed to complete this study, with all of them showing qualitatively similar features. All the figures and results summarized here refer then to the systematic study of one of the clones.

\section{Human SP-D (hSP-D)}


hSP-D was a kind gift from Dr. Paul Kingma (Cincinnati Children's Hospital, Cincinnati, OH). Human SP-D was obtained from bronchoalveolar lavages of human patients suffering from Pulmonary Alveolar Proteinosis (PAP), using protocols currently in use in the Hospital. Purification of hSP-D was done as described elsewhere [20].

\section{AFM sample preparation and imaging}

$20 \mu \mathrm{L}$ of protein solution at $0,85 \mathrm{ng} / \mu \mathrm{L}$ were incubated for $4-5$ minutes onto freshly cleaved mica. The surface was thoroughly rinsed with 6-7 mL of deionized water to remove buffer components. Finally, the sample was dried under nitrogen air flow [21].

Samples at acidic and basic $\mathrm{pH}$ were prepared in the same way, but $\mathrm{pH}$ was previously changed by dilution up to the final protein concentration $(0,85 \mathrm{ng} / \mu \mathrm{L})$, with citrate buffer (200 $\mathrm{mM} \mathrm{NaCl}, 20 \mathrm{mM}$ citrate, $1 \mathrm{mM}$ EDTA) when shifting to acid $\mathrm{pH}$ or Tris buffer (200 mM NaCl, $20 \mathrm{mM}$ Tris $\mathrm{pH} 8.5,1 \mathrm{mM}$ EDTA) for basic $\mathrm{pH}$. Protein was incubated at $4{ }^{\circ} \mathrm{C}$ during a minimum of 30 minutes before use.

Samples were scanned under an AFM from Nanotec (Nanotec Electrónica, Madrid, Spain) using PointProbePlus tips, type PPP-NCH (Nanosensors, Neuchâtel, Switzerland). Amplitude Modulation AFM (AM-AFM) mode was used for imaging in air, at room temperature and low humidity. Image processing (general plane subtraction, line flattening) and height profiles were done with WSxM freeware [22].

\section{Gel electrophoresis}

rhSP-D oligomeric forms were separated in 4-15\% polyacrylamide gels. Native electrophoresis was carried out in the absence of chemical denaturing agents and heating shock. Protein samples were prepared with loading buffer $(5 \mathrm{X})$ containing $5 \%$ Blue G-250 and 1 M 6-aminocaproic acid. Electrophoresis was performed at $4{ }^{\circ} \mathrm{C}$ at $110 \mathrm{~V}$ for 30 minutes, followed by 2 hours at $180 \mathrm{~V}$. [23]. For non-reducing conditions, Laemmli buffer was used as loading buffer [24] and for reducing conditions, $4 \% \beta$ mercaptoethanol was added. Samples were heated at $99{ }^{\circ} \mathrm{C}$ for 10 minutes prior to loading. Gels were developed by silver staining.

Protein cross-linking was performed with glutaraldehyde (GA) from MerckMillipore, at a final concentration of $0.5 \%$ or $1 \%(\mathrm{v} / \mathrm{v})$ for 2,15 or 30 minutes, 
according to a protocol modified from [25]. Reaction was stopped by adding nonreducing loading buffer and heating at $99{ }^{\circ} \mathrm{C}$ for 10 minutes.

For Western Blot (WB) analysis, proteins were transferred to PVDF membranes. rhSP-D was detected by a mouse anti-SP-D antibody. Horse anti-mouse IgG horseradish peroxidase-conjugated antibody was used as the secondary antibody. Protein bands were detected by chemiluminescence.

\section{De-glycosylation experiments}

Non-denatured rhSP-D was treated with the enzyme PNGase F (from NEB) following manufacturer protocol. The result was analysed by AFM and gel electrophoresis under non-reducing conditions, developed with silver staining as previously described.

\section{pH-dependent thermal stability experiments}

For protein stability experiments against thermal denaturation, rhSP-D was diluted to the final required concentration with citrate buffer at $\mathrm{pH} 4,2(200 \mathrm{mM} \mathrm{NaCl}$, $20 \mathrm{mM}$ citrate, $1 \mathrm{mM}$ EDTA) and incubated in ice for 30 minutes. Then, samples were heated at desired temperature $\left(35,40,45,50,55\right.$, or $\left.60{ }^{\circ} \mathrm{C}\right)$ for 15 minutes and crosslinked as described above. Electrophoresis was performed under non-reducing conditions. 


\section{RESULTS}

\section{Qualitative and quantitative analysis of rhSP-D oligomeric forms by AFM}

Atomic force microscopy of rhSP-D samples deposited onto mica surfaces at $\mathrm{pH}$ 7.4 revealed that rhSP-D is assembled in a variety of different oligomeric structures including trimers, hexamers, dodecamers, and larger aggregates (usually known as fuzzy balls, composed by variable number of trimers) (figure 1A). Dodecamer was the most abundant structure in this and other rhSP-D clones studied. Close details of the four different oligomeric species observed are shown in figures $1 \mathrm{~B}$ and $1 \mathrm{C}$.

To perform a quantitative analysis, a collection of high-quality images of $1 \mu \mathrm{m}-$ scan area was taken among different sample preparations and regions. Molecules were counted and classified in groups according to their oligomeric state. Table 1 summarizes the results obtained for a total of 660 molecules identified. A relative distribution of the different protein oligomers was calculated considering three different parameters: i) \% molecules, from the number of individual molecules within each oligomerization state, ii) \% weight, from the amount of total protein mass in each of the 4 groups considering the molecular weight of each oligomer, defined as 43,000 $\mathrm{Da} /$ monomer unit times the number of monomer units per oligomer; and iii) \% trimers, from the amount of trimers contained per oligomeric form group. According to these results, $41 \%$ of molecules and $51 \%$ of mass of rhSP-D is assembled as dodecamers, with dodecamers being the major constituent of this recombinant protein. The heterogeneous higher order oligomers ("fuzzy balls") are the second most abundant species (29\%) in mass, and trimers and hexamers constitute minor components, with $11 \%$ and $9 \%$, respectively, of protein mass.

\section{Structural characterization of rhSP-D oligomeric forms}

To further characterize the distinct oligomeric forms, we measured the position and size of relevant structural features from AFM height profiles of individual trimers, hexamers, dodecamers, and other higher-order structures (Figure 2).

- Trimers: rhSP-D trimer images in figure $2 \mathrm{~A}$ show the carbohydrate recognition domain with unprecedented resolution, enough to differentiate its three individual globular domains. Previous results published in the literature showed only a solid bright white spot at the end [26-28]. A bright protuberance was observed at $47 \pm 5$ $\mathrm{nm}$ from the C-terminal end, which likely corresponds to the $\mathrm{N}$-glycosylation site of 
the molecule, located at residue N70 in the collagen domain [14]. To prove that the rhSP-D used in these experiments was N-glycosylated, de-glycosylation experiments were carried out with PNGase F, which removes the sugar core attached to an asparagine residue through an $\mathrm{N}$-acetyl-glucosamine group (see Figure 3). A consistent shift in electrophoretic mobility was found between intact rhSP-D and PNGaseF-cleaved rhSP-D (figure 3A) accompanied by a disappearance of the bright protuberance in AFM images of the protein (figure $3 \mathrm{~B}$ ), confirming that rhSP-D is N-glycosylated. The AFM height profile of the trimer (figure 2A) additionally confirmed the three relevant features (numbered 1-3), corresponding to the $\mathrm{N}$-terminal end, the $\mathrm{N}$-glycosylation site and the $\mathrm{CRD}$, respectively. In addition, along the collagen domain we often observed several conspicuous, regularly spaced, bright segments. Resolution limitations precluded the unequivocal assignment of these bright spots to the collagen triple helical twists.

- Hexamers: rhSP-D hexamers are structures consisting of two trimers linked by the $\mathrm{N}$-terminal domain (figure $2 \mathrm{~B}$ ). Two populations of hexamers with different shapes were found: hexamers with a closed conformation (V-like) and hexamers with an open (stick-like) configuration, with the latter being the most abundant (as quantitated in Table 1). The presence of hexamers opens the possibility that these oligomers could be a structural intermediate between trimers and dodecameric forms, especially considering the way the N-terminal regions of dodecamers are seen under AFM. The distance between two opposed CRD in the hexamers contained in the molecule was $136 \pm 8 \mathrm{~nm}$, slightly longer than published data for rat SP-D $(114 \pm 3 \mathrm{~nm})$ [12]. Its symmetrical height profile exhibited the highest point in the middle of the molecule, which matches with the N-terminal domain of both hexamers constituting the interaction point (figure $2 \mathrm{~B}$ ).

- Dodecamers: rhSP-D dodecamer seems to be constituted by close association of two hexamers, although the part of the molecules that would be responsible for this interaction is not fully clear. The central junction had a radius of $12 \pm 2 \mathrm{~nm}$ and an apparent height of $1.0 \pm 0.4 \mathrm{~nm}$ as determined from the AFM profile (figure $2 \mathrm{C}$ ). In the dodecamers, it is quite frequent to observe a close apposition between $\mathrm{N}$ glycosylation sites from the two possible different hexamers at one or even at both sides of the $\mathrm{N}$-terminal domain (see supplementary figure $\mathrm{S} 4, \mathrm{C}$ and $\mathrm{F}$ ). It is not known whether this interaction could have an influence in protein oligomerization. 
An open question is whether "V-like" and "stick-like" hexamers might promote formation of different oligomeric structures.

- Higher order oligomers: Fuzzy balls are heterogeneous higher order oligomers constituted by a variable number of trimers, usually in a range between 6 and more than 20 , as it is observed in figure $2 \mathrm{D}$. They adopted a circular disposition once adsorbed onto the mica-substrate with a diameter similar to that of dodecamers. It is apparent that the center of fuzzy balls is not a solid unique junction, but a cluster of several small nearby centers, which could correspond to the N-terminal domain of trimers pre-associated in dodecamers or hexamers. The width and the height of the central structure increased with the number of trimers contained in the fuzzy balls, while the total diameter of the full complex remained constant (supplementary Figure S2). Most fuzzy-balls presented well-separated pairs of trimers around the central interacting region, suggesting that these structures are built from hexamers in their closed (V-like) conformation.

To confirm that the structures observed in the recombinant human SP-D sample produced by $\mathrm{CHO}$ cells are also present in the human protein obtained from natural sources, hSP-D purified from bronchoalveolar lavage of patients suffering from pulmonary alveolar proteinosis (PAP) was analyzed by AFM. PAP is a disease characterized by a dysregulation of surfactant production and metabolism,. It has been described in the literature that SP-D from PAP patients has higher amount of large oligomeric forms, as a consequence of this disease [29]. Figure 4 shows that hSP-D also consisted of multiple oligomeric forms including trimers, hexamers, dodecamers and fuzzy balls. Size and height of these structures were found to be similar to those in rhSP-D, with the most abundant species in SP-D from PAP patients being the heterogeneous higher order oligomeric (fuzzy balls) species. As a result, it was concluded that $\mathrm{CHO}$ cells represents a good system to produce rhSP-D protein in which the human SP-D oligomeric structures are reproduced. The exact quantitative distribution of different oligomeric forms was not calculated in the SP-D from PAP because these preparations were much less clean and it was often difficult to unequivocally assign each molecule to a defined oligomerization group.

\section{Electrophoretic analysis of rhSP-D}

In order to gain insight about the type of interactions leading to formation of different rhSP-D oligomers and to confirm the presence of the structures observed by 
AFM in the samples examined in bulk, a biochemical analysis using PAGE was conducted. Treatment of rhSP-D protein with $\beta \mathrm{ME}$ totally transformed all oligomeric species into monomeric form, as deduced by its electrophoretic behavior (figure $5 \mathrm{~A}$ ) due to the rupture of interchain disulfide bonds [11]. Under non-reducing conditions and in the presence of SDS the sample was found to consist mainly in trimers (figure $5 \mathrm{~A})$. Blue native electrophoresis was carried out to analyze protein native state. We expected to see protein bands principally at the theoretical mobilities corresponding the molecular weights of hexamer, dodecamer and at least smaller sized fuzzy balls in the gels, but the experiments revealed that under such conditions most of the protein remained in the well (figure $5 \mathrm{~B}$ ). It was necessary to add SDS to the sample to allow the protein to enter the gel, but rhSP-D was then observed primarily in the form of trimers (figure $5 \mathrm{~B}$ left and $5 \mathrm{C}$ ). In order to detect higher order oligomeric conformations, we sujected rhSP-D native structure to crosslinking by glutaraldehyde (GA), a bifunctional reagent that binds and interconnect neighbor amine groups at the protein. After 2 minutes of GA crosslinking reaction, PAGE performed under nonreducing conditions revealed protein bands with mobilities corresponding to hexameric and dodecameric structures, while only the dodecamer band was seen after 30 minutes of crosslinking reaction (figure 5A). Cross-linked samples were also subjected to native electrophoresis with a modification in the protocol, where the typical native running buffer was substituted by non-reducing running buffer (which contains SDS), in order to push the protein through the pores (figure $5 \mathrm{~B}$ right). No protein bands were observed in the gel when the experiment was carried out with cross-linked samples and native loading buffer (not shown).

\section{Effect of $\mathrm{pH}$ on the conformation and oligomerization of rhSP-D}

To further characterize the oligomerization pathway of SP-D and the type of interactions involved, the effect of acidic and basic $\mathrm{pH}$ on rhSP-D structure was studied. Protein was diluted to the desired concentration for AFM experiments with buffer at different pHs (8.5, 5.5, 4.2 and 3.0). AFM imaging of these samples was carried out followed by a quantitative analysis of the distribution of oligomeric forms (figure 6A). The results revealed that the proportion of protein assembled as dodecamers was higher in a more acidic environment, concomitant to a reduction in the percentage of fuzzy balls. We found that at $\mathrm{pH} 5.5$ and 4.4 there is a reduction of $10-15 \%$ by weight in the proportion of fuzzy balls compared to that at $\mathrm{pH} 7.4$, 
accompanied by an increase in dodecamers, but not in trimers (figure 6B). This supports the hypothesis that dodecamers are joined by weak interactions to create fuzzy balls, instead of being formed directly by covalent interactions between the trimers, with all of them linked together by the N-terminal end, a possibility previously suggested $[10,12]$. At $\mathrm{pH} 3$, the increase in dodecamer content was similar $(10 \%)$, but a higher decrease in fuzzy ball-like oligomers (25\%) was observed. This was accompanied in this case by a $9 \%$ increase in both trimers and hexamers, suggesting that at extremely low $\mathrm{pH}$, dodecamers could start to dissociate into hexamers and/or trimers. At basic $\mathrm{pH}(8.5)$, a reduction in the proportion of trimers and hexamers was observed, accompanied by an increase in dodecamer content -although not as high as observed in acidic environment- and in large oligomers (34\%). The content in fuzzyball-like aggregates seems to continuously increase from $\mathrm{pH} 3$ to $\mathrm{pH} 8.5$, indicating that $\mathrm{pH}$ alkalization promotes formation of large oligomers. Changes in the distribution of oligomers due to acidic $\mathrm{pH}$ were confirmed by electrophoresis, where rhSP-D samples at different pHs were crosslinked with GA (figure 6C). The most prominent dodecamer band is observed at $\mathrm{pH} 4.2$.

Interestingly, a conspicuous change in the conformation of dodecamers was clearly observed in the AFM images as a consequence of acidic $\mathrm{pH}$ (figure 6D). At the most acidic pHs (4.2 and 3.0) most of dodecameric molecules appeared in a sort of closed configuration, with the four trimers and the 4 trimeric CRD domains clustered in a nearby space. These cryptic structures appeared in all possible orientations, discarding any artifact from the washing and drying sample preparation procedures. At $\mathrm{pH} 5.5$, an intermediate state between those at $\mathrm{pH} 7.4$ and 4.2 was observed, with some dodecamers attaining a closed conformation and others in a standard X-like open conformation. In order to further characterize this structural conversion quantitatively, the four angles enclosed in each dodecamer were measured, data were pooled for each $\mathrm{pH}$ condition and represented as histograms (figure 7A, top). At $\mathrm{pH} 7.4$, the histogram was composed of two main angle populations, one corresponding to acute angles of $30^{\circ}-50^{\circ}$ and the other to obtuse angles of $140^{\circ}-150^{\circ}$, indicating that the dodecamers exhibit an X-like shape. Interestingly, the two acute angles were not identical in each X-like dodecamer. The ratio $\alpha_{1} / \alpha_{2}$ calculated for 273 dodecamers, where $\alpha_{1}$ is the smallest acute angle in the molecule and $\alpha_{2}$ the second smallest, peaked at 0.7 (see supplementary figure S3). This suggests that dodecamers are likely composed of two hexamers in open (stick-like) conformation as the assembly by two hexamers in closed conformation would have provided a value closer to 1.0. 
At pHs 4.2 and 3.0, the histograms of angles only show one main peak around acute angles of $20^{\circ}-40^{\circ}$ and a large dispersion of different values of obtuse angles. These data reflect how most dodecamers are in a closed conformation. At $\mathrm{pH}$ 5.5, an intermediate state is observed, where the peak of acute angles is present and the incidence of obtuse angles with values between $100^{\circ}$ to $180^{\circ}$ is still significant, but the peaks are not as prominent as observed at $\mathrm{pH}$ 7.4. This indicates that some dodecamers are changing to a closed conformation while others are still in a X-like conformation.

The angle formed by each hexamer (enclosed between its two trimers) was also measured and data was compiled in histograms (figure $7 \mathrm{~A}$, bottom) showing the same trend than observed in dodecamers.

Changes in conformation between $\mathrm{pH} 7.4$ and 4.2 were also studied in fuzzy balls. In this case, the parameters evaluated were the total molecule diameter and the center diameter. The results revealed that fuzzy balls at $\mathrm{pH} 4.2$ are characterized by a shorter total molecule diameter and larger center diameter than at $\mathrm{pH}$ 7.4. These results are in agreement with dodecamer conformational changes so that fuzzy balls are also taken to a close conformation at acidic $\mathrm{pH}$ (figure $7 \mathrm{~B}$ ).

\section{Thermal stability of rhSP-D at neutral and acidic pH}

The thermal stability of the structure of rhSP-D, especially of dodecamers, was examined by gel electrophoresis and AFM at $\mathrm{pH} 7.4$ and 4.2, and the results are shown in figure 8 . The structure of rhSP-D is more resistant to thermal denaturation in an acidic environment than at neutral $\mathrm{pH}$. At $\mathrm{pH} 4.2$ and heated to $40-45{ }^{\circ} \mathrm{C}$ it is still possible to observe bands in the gels corresponding to hexamers and dodecamers, while at $\mathrm{pH} 7.4$, the bands of these oligomeric forms are not clearly defined at the same temperatures. It seems that the conformational change occurring in the protein at $\mathrm{pH}$ 4.2 could make it more resistant to thermal denaturation. AFM imaging of rhSP-D at $\mathrm{pH} 7.4$ and $45{ }^{\circ} \mathrm{C}$ show how SP-D is dissembled and many triple collagen domains are unstructured (i.e. marked by a dotted circle in figure 8). In contrast, at the same temperature at $\mathrm{pH}$ 4.2, hexamers are the most abundant species observed in AFM images, followed by trimers, all of them constituted by properly assembled triple collagen helices (see figure 8, solid-line circles). AFM images therefore confirm that rhSP-D thermal stability is highly dependent on collagen thermal stability. 


\section{DISCUSSION}

Trimers, dodecamers and larger aggregates have been described as the different oligomeric forms of SP-D $[8,10,12,26,28]$. However, the actual distribution and function of these different forms still remain as open questions. The particular shape of dodecamers and the large aggregates known as 'fuzzy balls' constitutes a disadvantage in order to separate and quantify the different fractions [ 12 , 28, 30], electrophoresis experiments have failed to catch structures larger than trimers [12] and in electron microscopy, SP-D cannot be observed under-close-to-native conditions due to the fixing and shadowing treatment required for sample observation, always under vacuum [8, 12]. Considering all together, a quantitative analysis of the oligomeric distribution of SP-D under different conditions remains as a challenge. This has prevented a proper understanding of how the structural versatility of SP-D modulates its function under the constraints imposed by alveolar biology. In the current study, we have used AFM as a tool to analyze, qualitative and quantitatively, the structures of hSP-D and rhSP-D produced in CHO cells under close to native conditions. Previous AFM observations had provided a rather qualitative description of SP-D structure [27, 28]. In the current study we have found that hSP-D and rhSP$\mathrm{D}$ are a mixture of trimers, hexamers, dodecamers and larger aggregates. The variety and morphology of the structures observed are similar to those previously reported by transmission electron microscopy [8], except that the dodecamer is confirmed as the most abundant species in the rhSP-D studied here, and the so-called fuzzy balls as the most abundant species in hSP-D obtained from proteinosis lung lavages. Dodecamers had been already described as the most abundant oligomeric structure of rat SP-D and its recombinant version (rrSP-D) as examined by electron microscopy [12, 26].

However, it is relevant that the conspicuous presence of a population of hexamers in the previous studies $[8,27,28]$ was either ignored [27, 28], or considered as a possible artifact of the assembly of SP-D [8]. We propose here that the hexamer is likely a intermediate in dodecamer and "fuzzy ball" assembly. In the model proposed in figure 9, three monomers associate into trimers, which are stabilized by disulfide bonds, trimers form hexamers and these are paired into dodecamers. These two last structures could be associated into variable higher order oligomers, grouped under the common denomination of 'fuzzy balls'. We propose that the oligomerization process that take trimers to fuzzy balls is governed by different non-covalent interactions at the different oligomerization levels. 
The high-quality AFM images obtained in the current study have allowed observation of unprecedented structural determinants, including individual CRD domains in trimers, defined $\mathrm{N}$-glycosylation sites, collagen triple helical twists and the junction points around the N-terminal region in dodecamers and fuzzy balls. The repetitive observation of hexamers in different sample preparations and the fact that dodecamers observed on the surface of the mica seem to be composed of two hexamers (see supplementary figure S4), open the question as to what kind of interactions could be involved in the assembly of hexamers to form dodecamers. Moreover, the identification of segmented fuzzy ball centers, possibly corresponding to individual dodecamers and/or hexamers suggests that the assembly of higher order structures could be a reversible and dynamic process that might contribute to modulate SP-D function in response to local conditions within the cell or tissue under different physiopathological contexts.

Using truncated versions of SP-D, Zhang and colleagues showed that the neck domain is indispensable for trimer formation [31]. Based on a combination of proteolytic mapping, metabolic labeling, and thermal stability studies of mutant vs. wild type proteins, it was proposed that residues $\mathrm{C} 15$ and $\mathrm{C} 20$ at the amino terminal domain of SP-D are crucial to establish interchain covalent interactions, which then allow the assembly of trimers into higher order oligomers [10, 12, 33]. On the other hand, the role of the collagen domain in protein assembly is still not fully clear. Ogasawara and Volker showed that the absence of the collagen domain prevents a recombinant rat protein from forming higher order oligomers [30]; in contrast, Kingma and colleagues reported association of 4 trimers in a collagen-deleted SP-D mutant expressed in mice, attributing to the $\mathrm{N}$-terminal domain the leading role in the assembly of higher order oligomers [17]. The cross-linking protocol developed in the current work is a useful tool that could complement future studies to clarify this question.

Our results provide additional evidence on the oligomerization pathway of SPD. Gel electrophoresis experiments (figure 5) confirmed that rhSP-D monomers associate into trimers stabilized through disulfide bonds, which are reduced in the presence of $\beta \mathrm{ME}$ in agreement with previous published results [9, 26]. Trimers are the minimal structural unit observed in AFM images (figure 1). The length of the collagen domain as measured under AFM, $48 \pm 5 \mathrm{~nm}$, agrees well with its expected 
theoretical length, $51 \mathrm{~nm}$, considering a distance of $8.6 \AA$ per G-X-Y triplet [34, 35]. The position of the $\mathrm{N}$-glycosylation site relative to the beginning of the collagen domain was determined at $9.2 \pm 2.5 \mathrm{~nm}$, a bit shorter than the theoretical value of 12.9 $\mathrm{nm}$ that could be expected only considering the position of the glycosylation site in the sequence and standard geometric considerations of the collagen triple helix. This indicates that specific interactions and condensing effects may modulate the structure of the collagenic region at local scale.

The possible existence of SP-D hexamers was mentioned in only two previous published studies. Crouch and colleagues suggested the possible existence of paired SP-D trimers associated in overlapping antiparallel arrangements [12] and LethLarsen et al. found after collagenase digestion, a peptide of 15-16 $\mathrm{kDa}$ which dissociated in six polypeptide chains after reduction [14]. AFM images of rhSP-D (figures 1 and 2) and hSP-D (figure 4) show the conspicuous presence of hexamers constituted by two trimers joined through their N-terminal domains. Several evidences obtained from these images indicate that hexamers are a necessary intermediate state in the oligomerization process: i) the absence of Y-shaped structures formed by three trimers, ii) the conspicuous association of two hexamers in some dodecameric assemblies, which do not exhibit a unique solid central hub at the Nterminal region (see blue arrow in figure $2 \mathrm{C}$, and panels $\mathrm{B}$ and $\mathrm{D}$ of supplementary figure $\mathrm{S} 4$ ), and iii) the parallelism between angle values formed by the trimeric arms in hexamers and dodecamers (figure 7A).

The larger aggregates observed in SP-D samples, typically described as 'fuzzyball' assemblies, could be the result of the association of dodecamers and/or hexamers. These assemblies have been traditionally interpreted as the result of the coalescence of multiple trimeric arms with a nearly spherical geometry. However, our images illustrate how, once deposited onto mica, they adopt a conformation with the $\mathrm{N}$ terminal region protruding out from the mica. This supports the existence of specific interactions between the collagen segments close to the N-terminal domains of different hexamers and/or dodecamers. As a consequence, the large SP-D aggregates should not probably be seen as truly spherical arrangements but as wide arrangements of hexamers and dodecamers in a bouquet joined through the N-terminal ends. The assembly would therefore remind those of the other pulmonary collectin, the protein SP-A. The stability of the fuzzy balls could be weak compared to that of dodecamers, 
being more sensitive to environmental conditions such as $\mathrm{pH}$ (see figure 6), a probable consequence of the key role of ionic interactions in supratrimeric protein oligomerization. Interestingly, there is also a parallelism in the behavior of surfactant collectins SP-A and SP-D upon exposure to acidic $\mathrm{pH}$. A conformational change, from open-arms to a tight closed-structure with the collagen domains grouped in a "stemlike" region and a shorter diameter of the CRD domains, has been reported for SP-A as a function of changes in $\mathrm{pH}$ or the ionic environment [40-42].

We have observed by gel electrophoresis and AFM experiments that acidic $\mathrm{pH}-$ promoted changes in SP-D conformation include an increase in the thermal stability of the protein. Previous published data showed a dependence of collagen thermal stability on $\mathrm{pH}[38,39]$, suggesting that the higher stability of SP-D at $\mathrm{pH} 4.2$ could be associated with a higher stability of its collagenic segments. In fact, thermal denaturation of the protein as observed under AFM (see figure 8) seems to include a conspicuous denaturation of collagen triple helices, which are unstructured at $45{ }^{\circ} \mathrm{C}$ at $\mathrm{pH} 7.4$.

Why SP-D exists as different oligomeric forms and whether those forms respond to different functions remains as a question under debate. Considering that trimers, hexamers, dodecamers and fuzzy balls have been all observed here and in previous structural studies with protein from different sources [8, 12, 27, 28, 43], it seems reasonable to propose that SP-D exists as an equilibrium between these oligomers, with the hexamers playing a key role in the assembly process. This equilibrium could vary under defined conditions, which could somehow modulate some specific activities. It has been described that higher order multimers are more effective to promote influenza virus aggregation in vitro [8]. In the same line, structures larger than trimers, probably dodecamers and fuzzy balls, are required for binding to bacteria [28] and for lung surfactant homeostasis [9]. It is important to consider that the existence of a dynamic equilibrium between the different SP-D oligomeric forms may be part of intrinsic, functionally important, features that could contribute to differential activities in relevant physiopathological situations.

It is unknown whether the conformational change observed here under acidic conditions may have any functional implication. It has been published that $\mathrm{pH}$ in the lung fluid may become acidic under disease or inflammation [44], down to around 6.5. The effect of $\mathrm{pH}$ on SP-A function and structure has been studied [45, 46], but not much is known about its effect on SP-D. The closed conformation and higher 
proportion of dodecamers could be particularly important for the fate of the protein along secretory or endocytic pathways, where it may reach organelles with acidic $\mathrm{pH}$ (close to 5.5) [47]. Dodecamers with the closed conformation are somehow similar to fuzzy balls, described as the most active species against certain pathogens, suggesting that this conformational change could lead to a more active molecule with CRD grouped to simultaneously bind a higher number of ligands clustered at the same surface.

In summary, we have characterized the different oligomeric forms of a recombinant human SP-D, determined its quantitative distribution and confirmed the presence of those structures in native human SP-D from pulmonary alveolar proteinosis patients. We propose a model for SP-D assembly based on AFM images and PAGE-SDS experiments with chemical cross-linking. This model includes hexamers as an essential intermediate in the pathway to dodecamer assembly. The importance of environmental conditions such as $\mathrm{pH}$ on SP-D oligomerization and its three-dimensional configuration has been also demonstrated. Complementary experiments should determine whether other functionally important environmental factors such as the presence of $\mathrm{Ca}^{2+}$ or surfactant lipids also contribute to modulate the structure and conformation of SP-D. Further studies will aid to fully understand the role of hexamers in protein assembly, for instance, through the production of rhSP-D truncated versions where some amino acids of the collagen domain could be deleted or mutated. The complementary functional characterization of mutated/truncated SP-D forms differing in supramolecular structure will complete our view of the importance of this collectin in lung homeostasis. 


\section{ACKNOWLEDGEMENTS}

This work has been supported by grants from MINECO, the Spanish Ministry of Economy (BIO2015-67930-R) and the Regional Government of Madrid (S2013/MlT2807). R.A. acknowledges a FPU fellowship from the Spanish Ministry of Education.

A.M.-G. acknowledges support from a FPI fellowship associated to project FIS201458328-P granted to F.M.-H. by Spanish MINECO. F.M.-H. also acknowledges support from European Research Council (ERC) under the European Union's Horizon2020 Research and Innovation Program (grant agreement No 681299).

\section{AUTHORS CONTRIBUTIONS}

R.A., J.R. and J.P.-G. designed the study. R.A., A.J., W.H.B., and A.M-G. performed experiments. R.A., M.E., J.R., F. M.-H. And J.P.-G. designed experiments and analysed data. J.R., M.E., F.M.-H. and J.P.-G. provided scientific advice and helped writing the manuscript. All authors interpreted and discussed the data, reviewed the manuscript and had final approval of the submitted version. 


\section{REFERENCES}

[1] Kishore U, Greenhough TJ, Waters P, Shrive AK, Ghai R, Kamran MF, et al. Surfactant proteins SP-A and SP-D: structure, function and receptors. Mol Immunol. 2006;43:1293-315.

[2] Sano H, Kuroki Y. The lung collectins, SP-A and SP-D, modulate pulmonary innate immunity. Mol Immunol. 2005;42:279-87.

[3] Kingma PS, Whitsett JA. In defense of the lung: surfactant protein A and surfactant protein D. Curr Opin Pharmacol. 2006;6:277-83.

[4] Crouch EC. Surfactant protein-D and pulmonary host defense. Respir Res. 2000;1:93-108.

[5] Persson A, Rust K, Chang D, Moxley M, Longmore W, Crouch E. CP4: a pneumocytederived collagenous surfactant-associated protein. Evidence for heterogeneity of collagenous surfactant proteins. Biochemistry. 1988;27:8576-84.

[6] Persson A, Chang D, Rust K, Moxley M, Longmore W, Crouch E. Purification and biochemical characterization of CP4 (SP-D), a collagenous surfactant-associated protein. Biochemistry. 1989;28:6361-7.

[7] Ikegami M, Carter K, Bishop K, Yadav A, Masterjohn E, Brondyk W, et al. Intratracheal recombinant surfactant protein d prevents endotoxin shock in the newborn preterm lamb. Am J Respir Crit Care Med. 2006;173:1342-7.

[8] Hartshorn K, Chang D, Rust K, White M, Heuser J, Crouch E. Interactions of recombinant human pulmonary surfactant protein $D$ and SP-D multimers with influenza A. Am J Physiol. 1996;271:L753-62.

[9] Zhang L, Ikegami M, Crouch EC, Korfhagen TR, Whitsett JA. Activity of pulmonary surfactant protein-D (SP-D) in vivo is dependent on oligomeric structure. J Biol Chem. 2001;276:19214-9.

[10] Brown-Augsburger P, Chang D, Rust K, Crouch EC. Biosynthesis of surfactant protein $\mathrm{D}$. Contributions of conserved $\mathrm{NH} 2$-terminal cysteine residues and collagen helix formation to assembly and secretion. J Biol Chem. 1996;271:18912-9.

[11] Crouch EC. Structure, biologic properties, and expression of surfactant protein D (SP-D). Biochim Biophys Acta. 1998;1408:278-89.

[12] Crouch E, Persson A, Chang D, Heuser J. Molecular structure of pulmonary surfactant protein D (SP-D). J Biol Chem. 1994;269:17311-9.

[13] Shrive AK, Tharia HA, Strong P, Kishore U, Burns I, Rizkallah PJ, et al. Highresolution structural insights into ligand binding and immune cell recognition by human lung surfactant protein D. J Mol Biol. 2003;331:509-23.

[14] Leth-Larsen R, Holmskov U, Hojrup P. Structural characterization of human and bovine lung surfactant protein D. Biochem J. 1999;343 Pt 3:645-52.

[15] Kuroki Y, Gasa S, Ogasawara Y, Shiratori M, Makita A, Akino T. Binding specificity of lung surfactant protein SP-D for glucosylceramide. Biochem Biophys Res Commun. 1992;187:963-9.

[16] Ogasawara Y, Kuroki Y, Akino T. Pulmonary surfactant protein D specifically binds to phosphatidylinositol. J Biol Chem. 1992;267:21244-9.

[17] Kingma PS, Zhang L, Ikegami M, Hartshorn K, McCormack FX, Whitsett JA. Correction of pulmonary abnormalities in Sftpd-/- mice requires the collagenous domain of surfactant protein D. J Biol Chem. 2006;281:24496-505. 
[18] Korfhagen TR, Sheftelyevich V, Burhans MS, Bruno MD, Ross GF, Wert SE, et al. Surfactant protein-D regulates surfactant phospholipid homeostasis in vivo. J Biol Chem. 1998;273:28438-43.

[19] Cairns VR, DeMaria CT, Poulin F, Sancho J, Liu P, Zhang J, et al. Utilization of nonAUG initiation codons in a flow cytometric method for efficient selection of recombinant cell lines. Biotechnol Bioeng. 2011;108:2611-22.

[20] Stahlman MT, Gray ME, Hull WM, Whitsett JA. Immunolocalization of surfactant protein-D (SP-D) in human fetal, newborn, and adult tissues. J Histochem Cytochem. 2002;50:651-60.

[21] Lyubchenko YL, Shlyakhtenko LS. AFM for analysis of structure and dynamics of DNA and protein-DNA complexes. Methods. 2009;47:206-13.

[22] Horcas I, Fernandez R, Gomez-Rodriguez JM, Colchero J, Gomez-Herrero J, Baro AM. WSXM: a software for scanning probe microscopy and a tool for nanotechnology. Rev Sci Instrum. 2007;78:013705.

[23] Schagger H. Blue-native gels to isolate protein complexes from mitochondria. Methods Cell Biol. 2001;65:231-44.

[24] Laemmli UK. Cleavage of structural proteins during the assembly of the head of bacteriophage T4. Nature. 1970;227:680-5.

[25] Leitner A, Walzthoeni T, Kahraman A, Herzog F, Rinner O, Beck M, et al. Probing native protein structures by chemical cross-linking, mass spectrometry, and bioinformatics. Mol Cell Proteomics. 2010;9:1634-49.

[26] Crouch E, Chang D, Rust K, Persson A, Heuser J. Recombinant pulmonary surfactant protein D. Post-translational modification and molecular assembly. J Biol Chem. 1994;269:15808-13.

[27] Hartshorn KL, Webby R, White MR, Tecle T, Pan C, Boucher S, et al. Role of viral hemagglutinin glycosylation in anti-influenza activities of recombinant surfactant protein D. Respir Res. 2008;9:65.

[28] Leth-Larsen R, Garred P, Jensenius H, Meschi J, Hartshorn K, Madsen J, et al. A common polymorphism in the SFTPD gene influences assembly, function, and concentration of surfactant protein D. J Immunol. 2005;174:1532-8.

[29] Crouch E, Persson A, Chang D. Accumulation of surfactant protein D in human pulmonary alveolar proteinosis. Am J Pathol. 1993;142:241-8.

[30] Ogasawara Y, Voelker DR. The role of the amino-terminal domain and the collagenous region in the structure and the function of rat surfactant protein D. J Biol Chem. 1995;270:19052-8.

[31] Zhang P, McAlinden A, Li S, Schumacher T, Wang H, Hu S, et al. The aminoterminal heptad repeats of the coiled-coil neck domain of pulmonary surfactant protein $d$ are necessary for the assembly of trimeric subunits and dodecamers. J Biol Chem. 2001;276:19862-70.

[32] Hoppe HJ, Barlow PN, Reid KB. A parallel three stranded alpha-helical bundle at the nucleation site of collagen triple-helix formation. FEBS Lett. 1994;344:191-5.

[33] Brown-Augsburger P, Hartshorn K, Chang D, Rust K, Fliszar C, Welgus HG, et al. Site-directed mutagenesis of Cys-15 and Cys-20 of pulmonary surfactant protein $D$. Expression of a trimeric protein with altered anti-viral properties. J Biol Chem. 1996;271:13724-30.

[34] Okuyama K. Revisiting the molecular structure of collagen. Connect Tissue Res. 2008;49:299-310. 
[35] Beck K, Brodsky B. Supercoiled protein motifs: the collagen triple-helix and the alpha-helical coiled coil. J Struct Biol. 1998;122:17-29.

[36] Persikov AV, Ramshaw JA, Brodsky B. Prediction of collagen stability from amino acid sequence. J Biol Chem. 2005;280:19343-9.

[37] Persikov AV, Ramshaw JA, Kirkpatrick A, Brodsky B. Electrostatic interactions involving lysine make major contributions to collagen triple-helix stability.

Biochemistry. 2005;44:1414-22.

[38] Freudenberg U, Behrens SH, Welzel PB, Muller M, Grimmer M, Salchert K, et al. Electrostatic interactions modulate the conformation of collagen I. Biophys J. 2007;92:2108-19.

[39] Bianchi E, Conio G, Ciferri A, Puett D, Rajagh L. The role of $\mathrm{pH}$, temperature, salt type, and salt concentration on the stability of the crystalline, helical, and randomly coiled forms of collagen. J Biol Chem. 1967;242:1361-9.

[40] Ridsdale RA, Palaniyar N, Holterman CE, Inchley K, Possmayer F, Harauz G. Cationmediated conformational variants of surfactant protein A. Biochim Biophys Acta. 1999;1453:23-34.

[41] Efrati H, Hawgood S, Williams MC, Hong K, Benson BJ. Divalent cation and hydrogen ion effects on the structure and surface activity of pulmonary surfactant. Biochemistry. 1987;26:7986-93.

[42] Palaniyar N, Ikegami M, Korfhagen T, Whitsett J, McCormack FX. Domains of surfactant protein $A$ that affect protein oligomerization, lipid structure and surface tension. Comp Biochem Physiol A Mol Integr Physiol. 2001;129:109-27.

[43] van Eijk $\mathrm{M}$, van de Lest $\mathrm{CH}$, Batenburg JJ, Vaandrager $\mathrm{AB}$, Meschi J, Hartshorn $\mathrm{KL}$, et al. Porcine surfactant protein $\mathrm{D}$ is $\mathrm{N}$-glycosylated in its carbohydrate recognition domain and is assembled into differently charged oligomers. Am J Respir Cell Mol Biol. 2002;26:739-47.

[44] Ng AW, Bidani A, Heming TA. Innate host defense of the lung: effects of lung-lining fluid pH. Lung. 2004;182:297-317.

[45] Ruano ML, Nag K, Casals C, Perez-Gil J, Keough KM. Interactions of pulmonary surfactant protein A with phospholipid monolayers change with $\mathrm{pH}$. Biophys $\mathrm{J}$. 1999;77:1469-76.

[46] Ruano ML, Perez-Gil J, Casals C. Effect of acidic $\mathrm{pH}$ on the structure and lipid binding properties of porcine surfactant protein A. Potential role of acidification along its exocytic pathway. J Biol Chem. 1998;273:15183-91.

[47] Demaurex N. pH Homeostasis of cellular organelles. News Physiol Sci. 2002;17:1-5. [48] Sato A, Whitsett JA, Scheule RK, Ikegami M. Surfactant protein-d inhibits lung inflammation caused by ventilation in premature newborn lambs. Am J Respir Crit Care Med. 2010;181:1098-105. 
TABLE 1

Quantitative distribution of different oligomeric forms of SP-D as determined from individual molecules observed under AFM at pH 7.4

\begin{tabular}{|c|c|c|c|c|c|c|c|}
\hline $\begin{array}{c}\text { Oligomeric } \\
\text { Form }\end{array}$ & No Molecules & \% Molecules & Weight (MDa) & Weight (g) & \% Weight & No Trimers & \% Trimers \\
\hline Total & 660 & 100 & 278.2 & $4.62 \mathrm{E}-16$ & 100 & 2169 & 100 \\
Trimer & 244 & 37 & 31.5 & $5.23 \mathrm{E}-17$ & 11 & 244 & 11 \\
Hexamer & 100 & 15 & 25.8 & $4.28 \mathrm{E}-17$ & 9 & 200 & 9 \\
Dodecamer & 273 & 41 & 140.9 & $2.34 \mathrm{E}-16$ & 51 & 1092 & 50 \\
Fuzzy Ball & 45 & 7 & 80.1 & $1.33 \mathrm{E}-16$ & 29 & 633 & 29 \\
\hline
\end{tabular}

The Weight (MDa), \%Molecules, and \%Weight are defined as described in Results. Weight $(\mathrm{g})$ is defined as Weight in Daltons/Avogadro's Number. 


\section{LEGENDS TO FIGURES}

\section{Figure 1}

\section{AFM images of rhSP-D at $\mathrm{pH} 7.4$}

$A-C$ : AFM images of rhSP-D, where illustrative examples of different oligomeric forms can be observed, including trimers $(\mathrm{T})$, hexamers $(\mathrm{H})$, dodecamers $(\mathrm{D})$, and fuzzy balls (FB). $A, 1 \mu \mathrm{m}$ scan, scale bar $200 \mathrm{~nm} . B$ and $C, 300 \mathrm{~nm}$ scan, scale bar 60 nm.

\section{Figure 2}

\section{Structural analysis of the different rhSP-D oligomeric forms}

At the first column, a scheme illustrates each type of oligomer that has been analyzed, with some characteristic parameters calculated from the AFM images. The position of the glycosylation site is indicated by Y. Parameters were calculated after measuring and averaging 50 individual molecules, and the average and its standard deviation is shown. The second column shows illustrative AFM images from each type of SP-D oligomer, scale bar: $30 \mathrm{~nm}$. Yellow arrows mark the bright spot at the N-glycosylation site (N170). The blue arrow points to the segment overlapping two clearly distinct hexamers at a dodecamer. The third column includes the height profiles taken from each oligomer along the green line drawn in the images of the fourth column. The numbers above the profile indicate 1: N-terminal domain, 2: $\mathrm{N}$-glycosylation site; 3: CRD.

\section{Figure 3}

\section{De-glycosylation of rhSP-D}

Panel A shows AFM images of trimers, hexamers and dodecamers before (-) and after (+) PNGase F treatment. Yellow arrow indicates the N-glycosylation site seen as a bright protrusion in the glycosylated protein. B, silver staining of gel with rhSP-D cleaved (+) or not (-) by PNGase F (indicated in above the gel). P: PNGase F. Scale bar: $25 \mathrm{~nm}$.

\section{Figure 4}




\section{AFM images of natural hSP-D isolated from PAP patients}

Human SP-D obtained from PAP patients has been analyzed under AFM. Three different scan areas where imaged: from left to right $1 \mu \mathrm{m}$, and $450 \mathrm{~nm}$. Presence of trimers $(\mathrm{T})$, hexamers $(\mathrm{H})$, dodecamers $(\mathrm{D})$ and fuzzy balls $(\mathrm{FB})$ has been highlighted.

\section{Figure 5}

\section{Biochemical analysis of rhSP-D by electrophoresis}

The quaternary structure of rhSP-D has been analyzed by PAGE-SDS ( $A$ and $C$ ) and native electrophoresis $(B)$. Above the gels the different conditions and reagents used to treat each sample are indicated. Panel A: the gel on the left has been developed by silver staining, the gels on the right side are WB revealed with anti-SP-D antibody. In panel $B$, the gel on the left is an anti-SP-D WB while the gel on the right has been revealed by silver staining. Gel in Panel $C$ is a WB. SDS* means that SDS was added to the sample before crosslinking with GA. The molecular weight of the standard proteins is indicated on the left of the gels.

\section{Figure 6}

\section{Effect of $\mathrm{pH}$ on the conformation and oligomeric distribution of rhSP-D}

Illustrative AFM images taken from rhSP-D samples at the different $\mathrm{pHs}$ indicated in the images; scale bar: $200 \mathrm{~nm}$ (A). Percentage by mass of the different oligomeric forms of rhSP-D at the different pHs (B). Electrophoretic behavior of the protein at the different $\mathrm{pHs}$ tested, once the samples were crosslinked with GA $0.5 \%$ for 15 minutes (C). Different symbols indicate the different types of oligomers: fuzzy balls (open triangle), dodecamer (triangle), hexamer (star) and trimer (circle). Details of dodecamers incubated at different $\mathrm{pH}$ conditions $(D)$.

\section{Figure 7}

Effect of $\mathrm{pH}$ on the configuration of rhSP-D dodecamers evaluated from the angles defined by their trimeric arms

A, the four angles enclosed by the trimeric arms within each dodecamer (A, top) were measured, pooled and their relative frequencies represented with a histogram at each 
of the different $\mathrm{pHs}$ evaluated. Inside each of the graphs a representative picture of a dodecamer with the typical configuration at the corresponding $\mathrm{pH}$ has been included, with the angles measured marked. "n" is the total number of angles measured for each condition. The same quantification was carried out for the angle enclosed by the two trimers in the hexamers (A, bottom). B, the frequency distribution of the center diameter (N-terminal domain) and the total molecule diameter is represented at $\mathrm{pH} 7.4$ (top panels) and 4.2 (bottom panels). The histograms have been fitted to a Gaussian distribution. A representative AFM image of fuzzy ball at each $\mathrm{pH}$ condition is showed on the right.

\section{Figure 8}

\section{Thermal denaturation of rhSP-D}

Top, a PAGE-SDS gel followed by western blot of rhSP-D subjected to an increasing gradient of temperatures, at $\mathrm{pH} 7.4$ or 4.2 , and then crosslinked as described in the methods section. The $\mathrm{pH}$ and temperature of treatment of each sample are indicated at the top of the WB membrane, the assigned oligomeric states of each band, on the right, and the molecular weight of standards, on the left. Bottom, representative AFM images of rhSP-D after incubation at $45^{\circ}$ at $\mathrm{pH} 4.2$ (left) and 7.4 (right). Structures enclosed in white solid circles are examples of trimers preserving a native structure, while structures enclosed in dotted circles are disassembled trimers due to thermal stress. Scale bar: $200 \mathrm{~nm}$.

\section{Figure 9}

\section{Proposed model for rhSP-D oligomerization pathway}

Three monomers of rhSP-D first associate through the folding of the collagenous sequence segment into triple helices and the assembly of a coiled-coil bundle of $\alpha$ helices in the neck region. These trimers are stabilized by two disulfide bonds in the cysteine-rich N-terminal domain. Two trimers would then form hexamers with the participation of non-covalent interactions, such as ionic interactions, between the two N-terminal domains. Next step would be the association of two hexamers into dodecamers by ionic and hydrophobic interactions, which could present sugars from opposed hexamers to interact, at one or both sides of the N-terminal domain. Fuzzy balls would be formed by association of dodecamers and/or hexamers through weaker non-covalent interactions, specially, hydrophobic interactions. 
At extreme acidic $\mathrm{pH}$, the configuration of the hexamers, dodecamers and fuzzy balls changes from open to closed, which could influence the whole assembly pathway because it is accompanied by an increase in the proportion of dodecamers and a decrease in amount of fuzzy balls. 
FIGURE 1
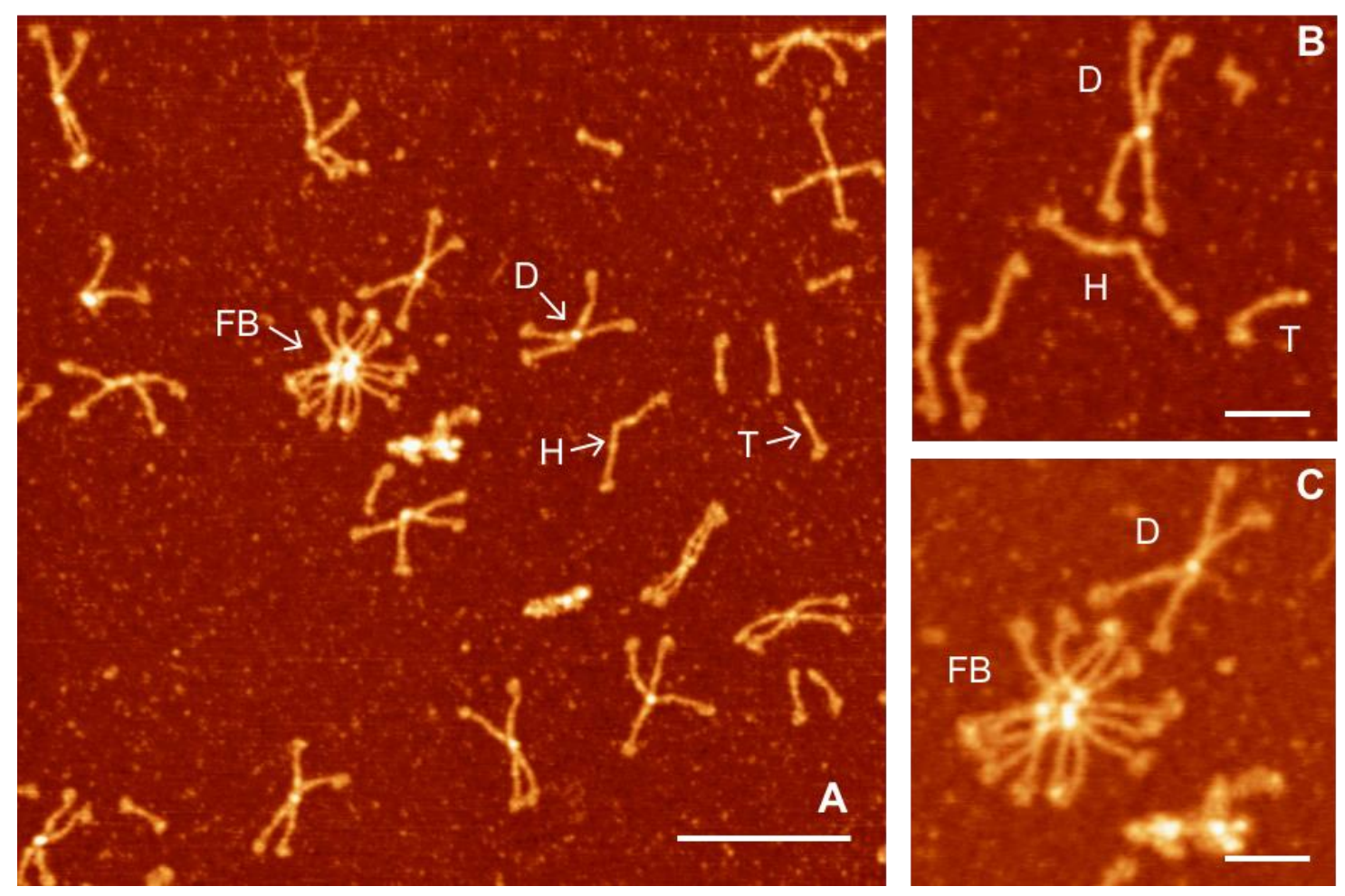


\section{FIGURE 2}

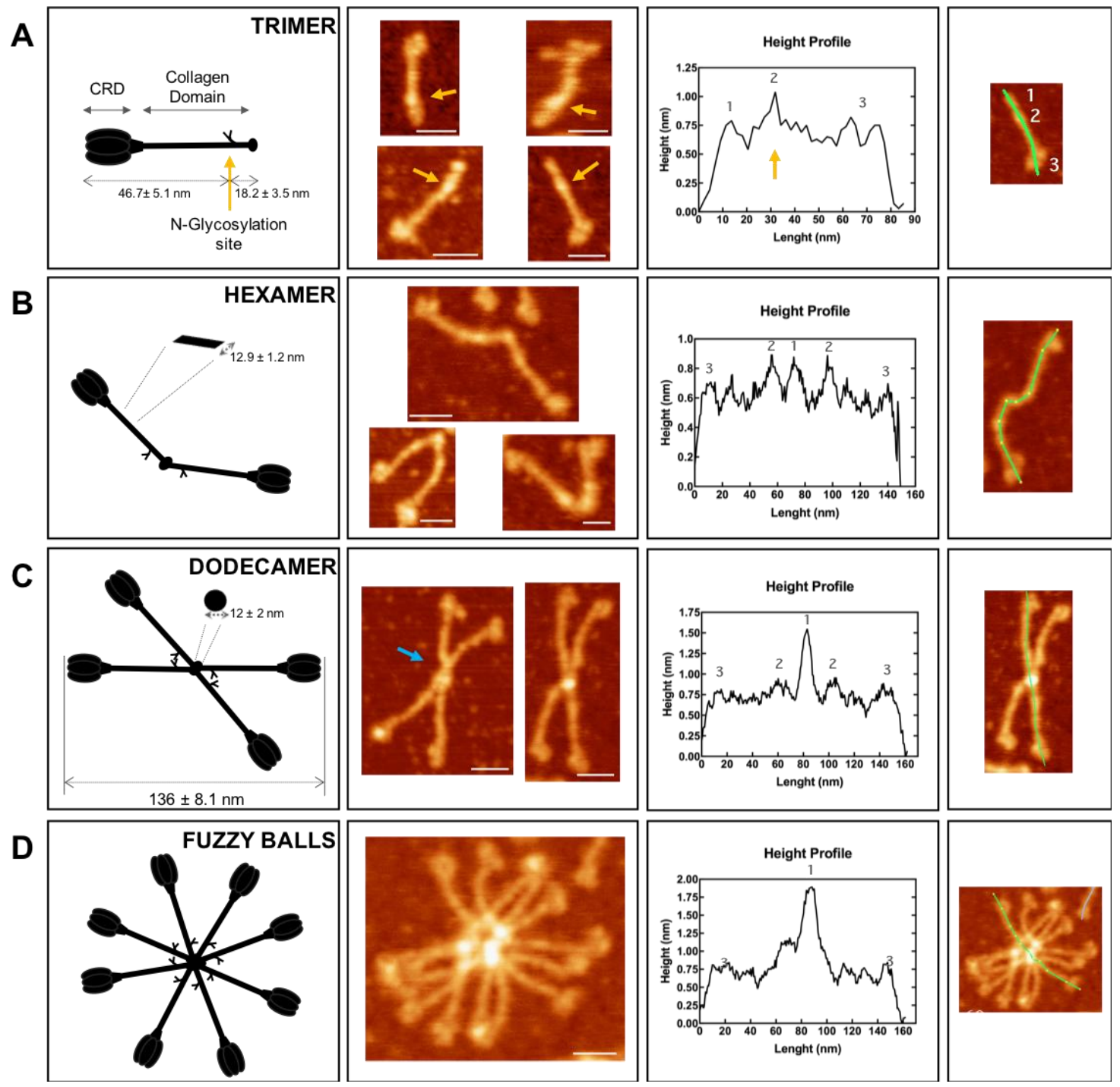


FIGURE 3

A

B

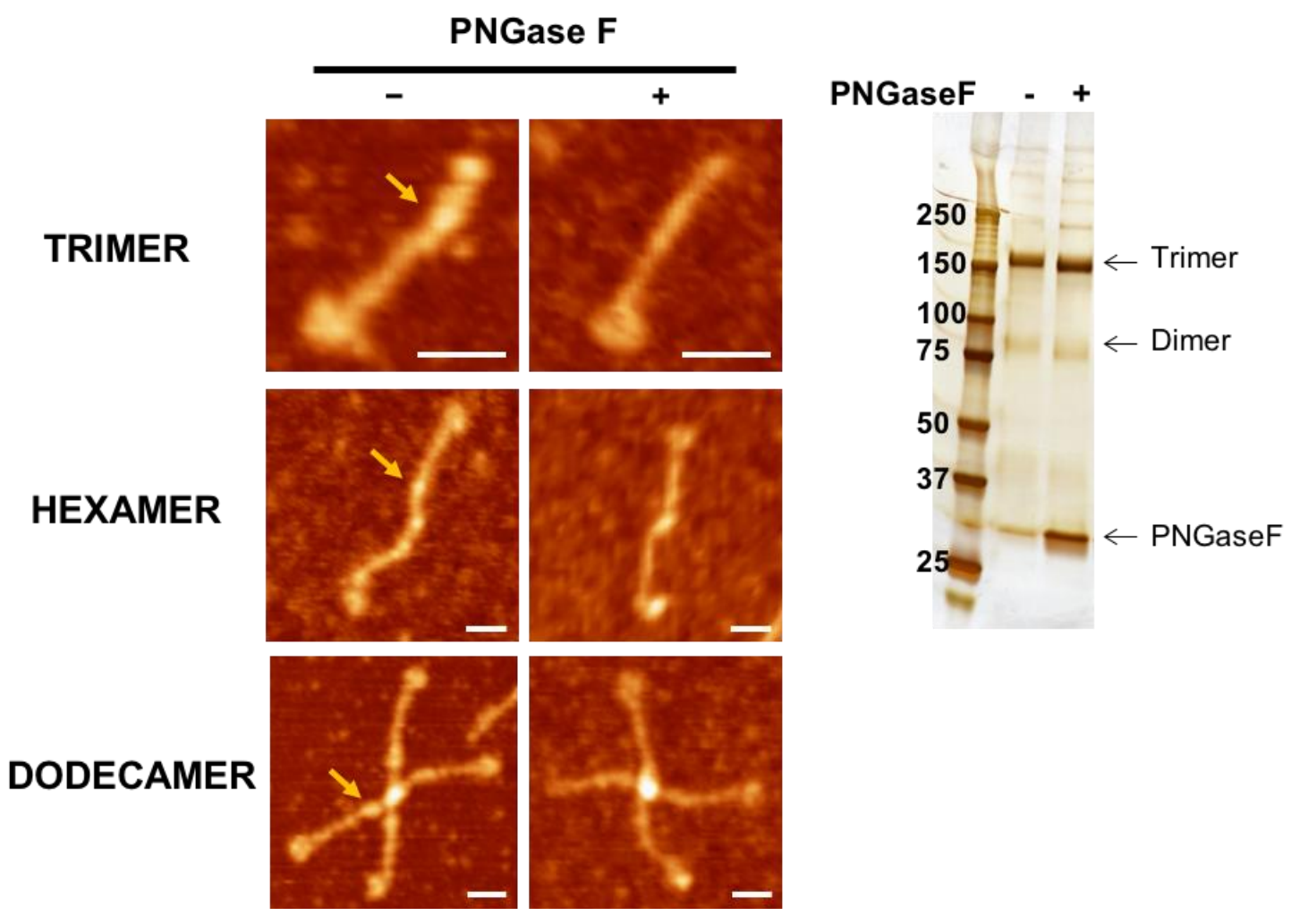


FIGURE 4

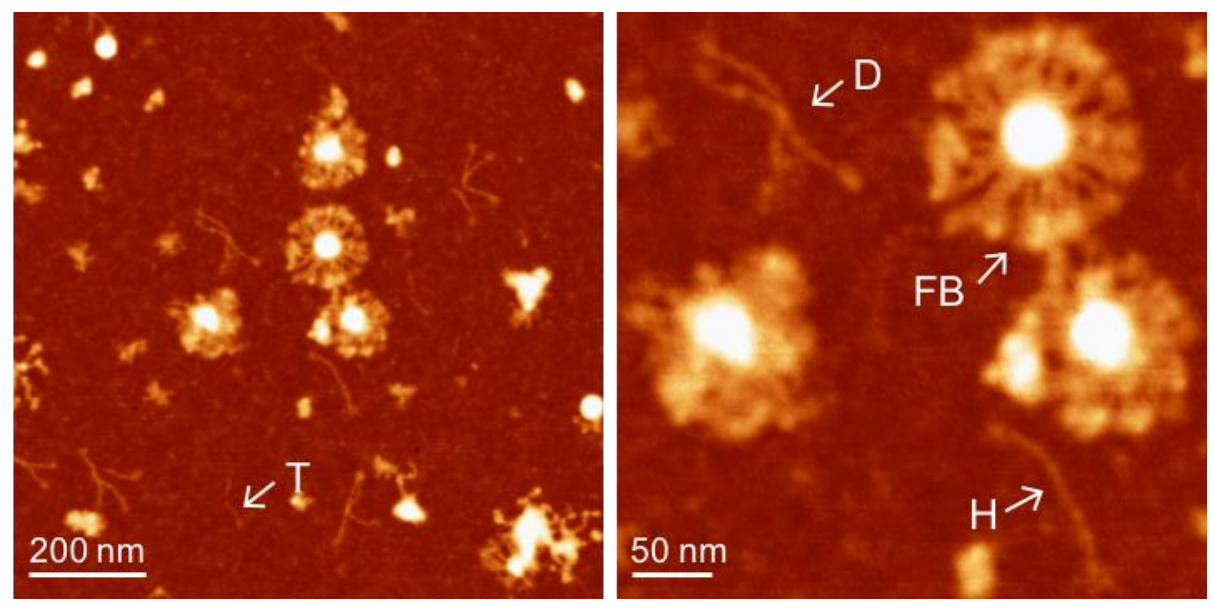




\section{FIGURE 5}
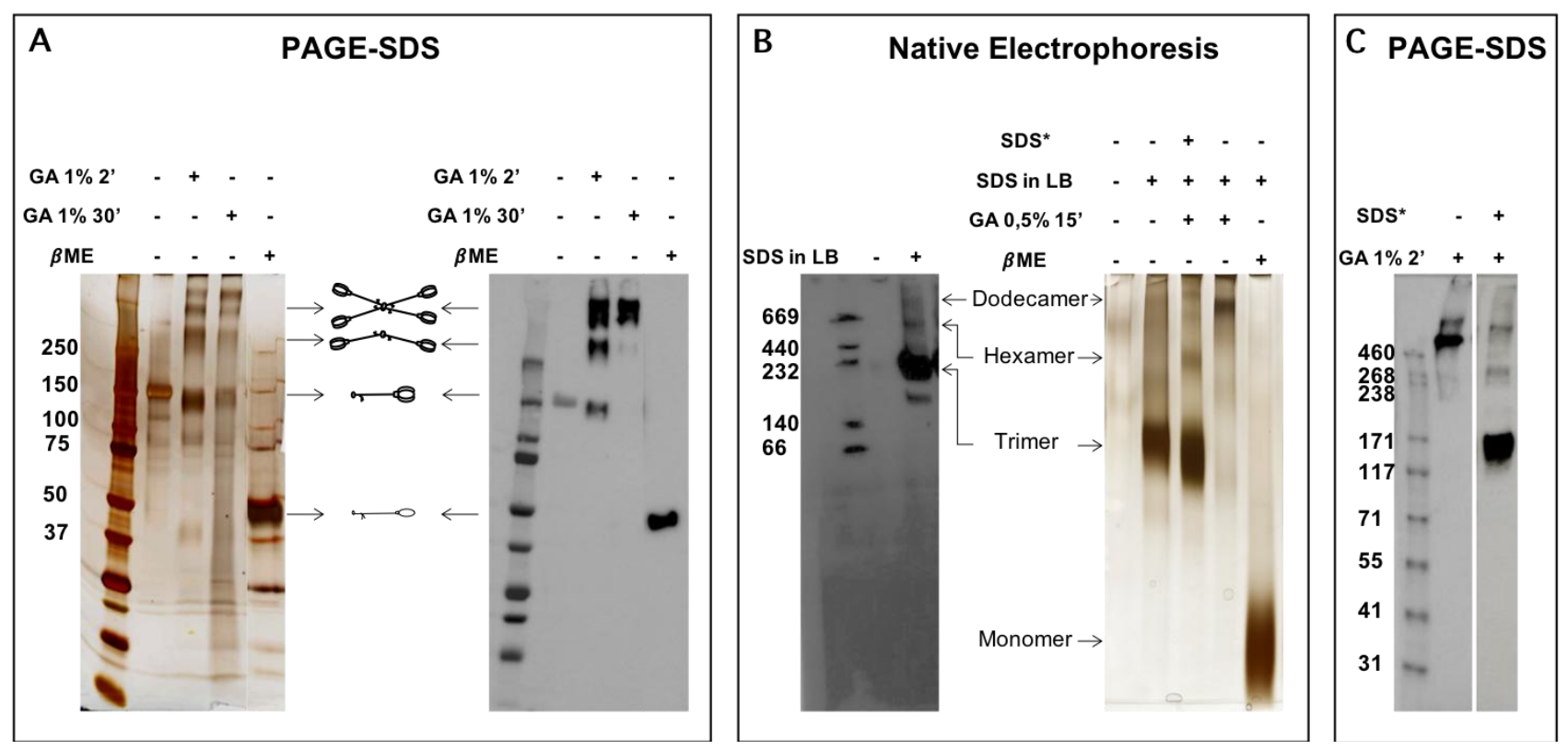


\section{FIGURE 6}

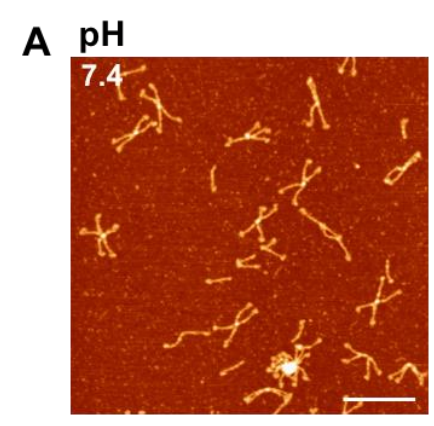

B

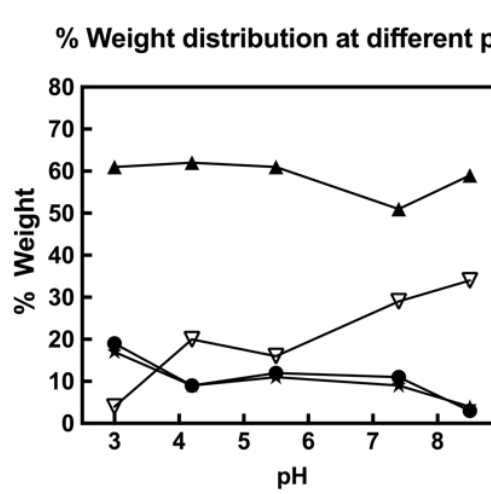

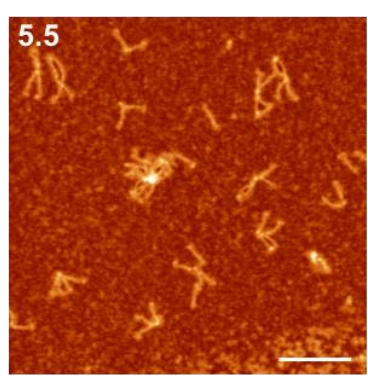
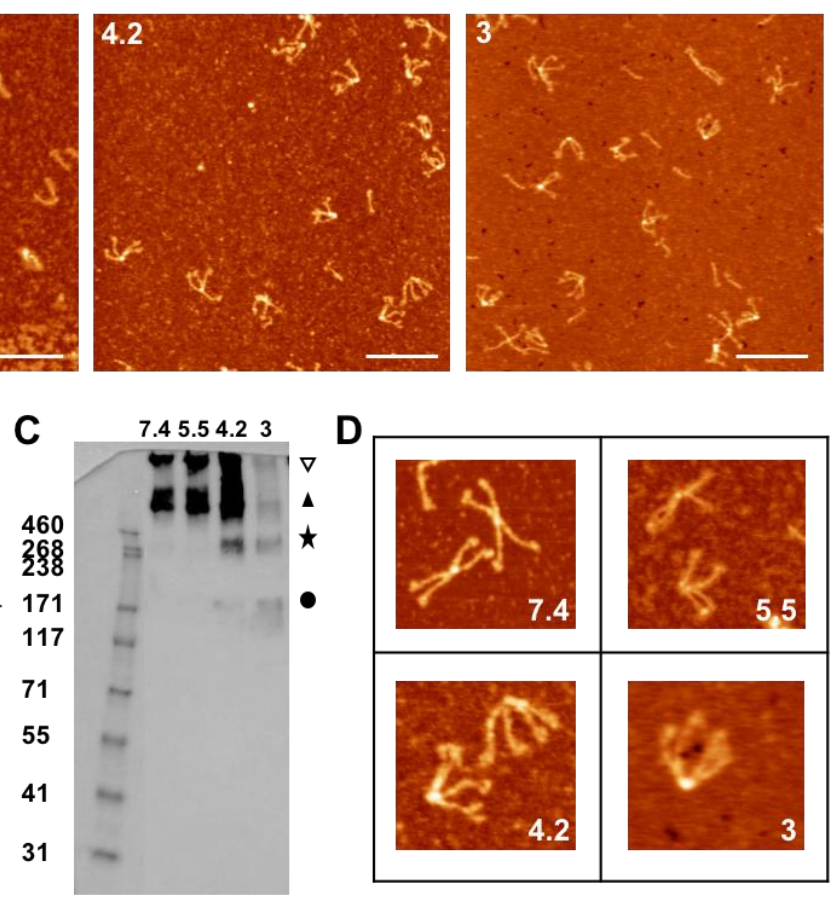


\section{FIGURE 7}

A

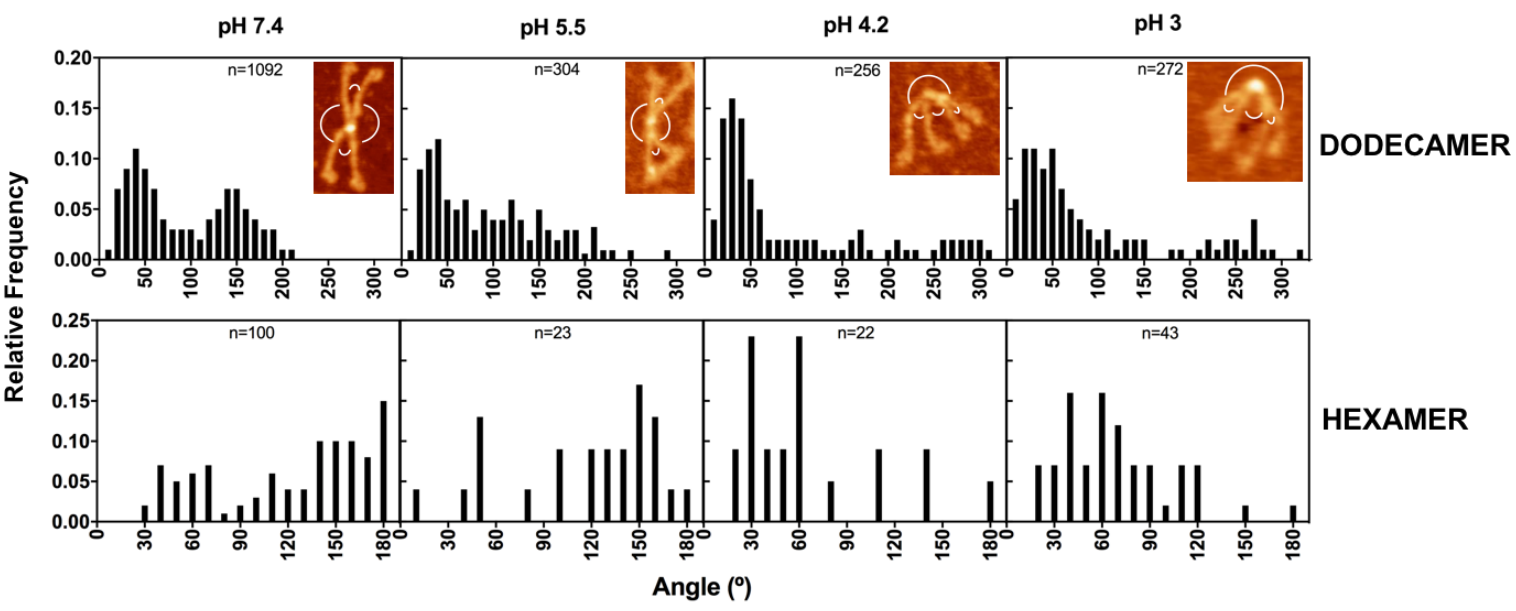

B
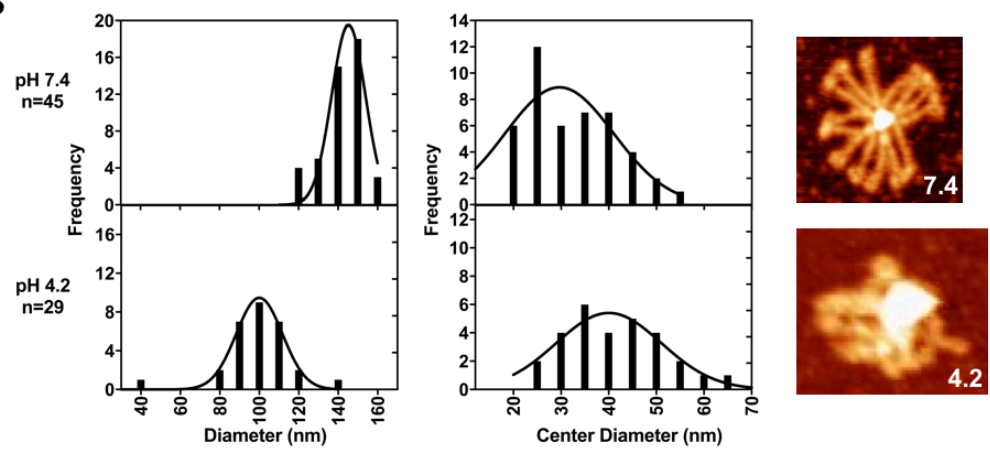


\section{FIGURE 8}

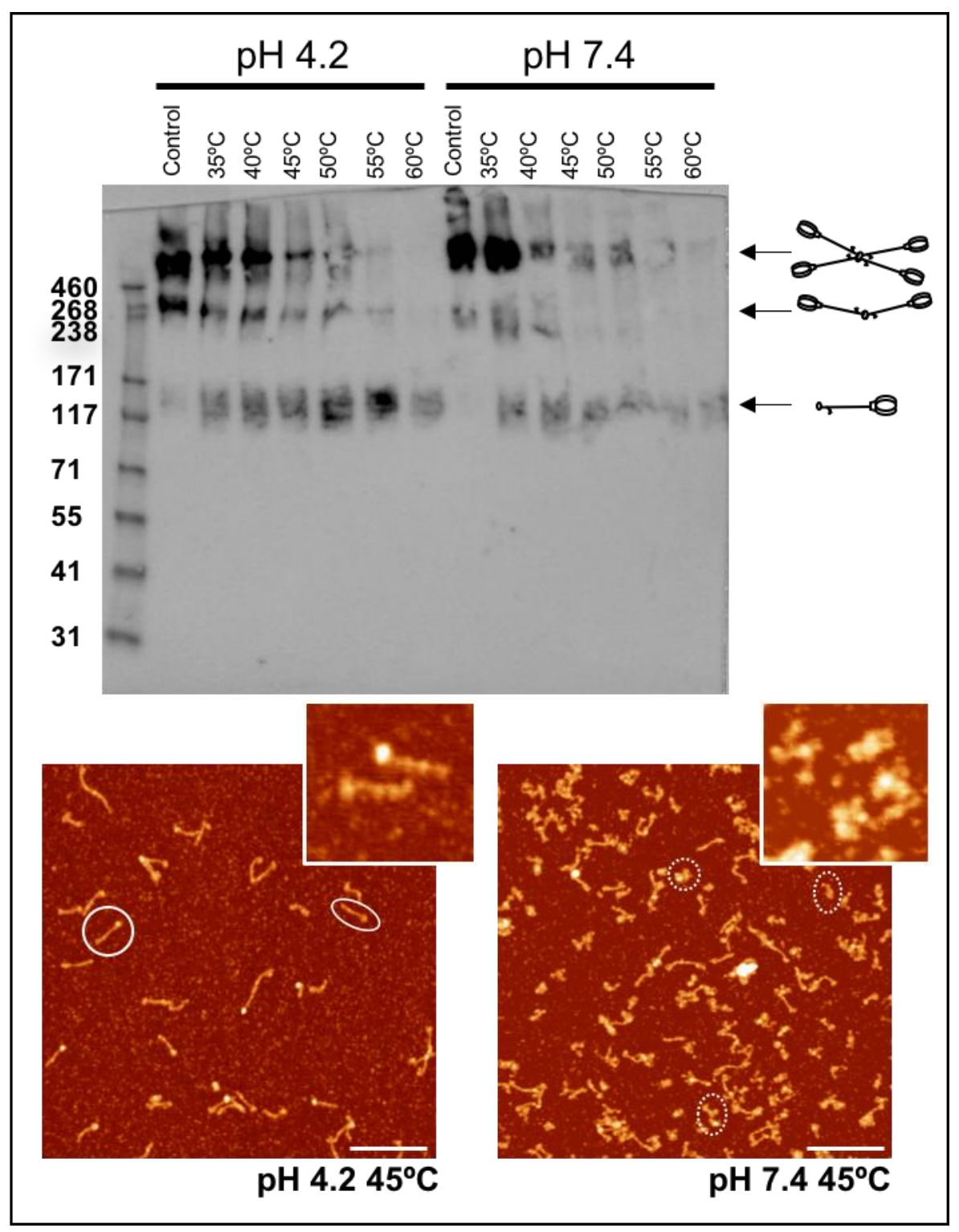




\section{FIGURE 9}
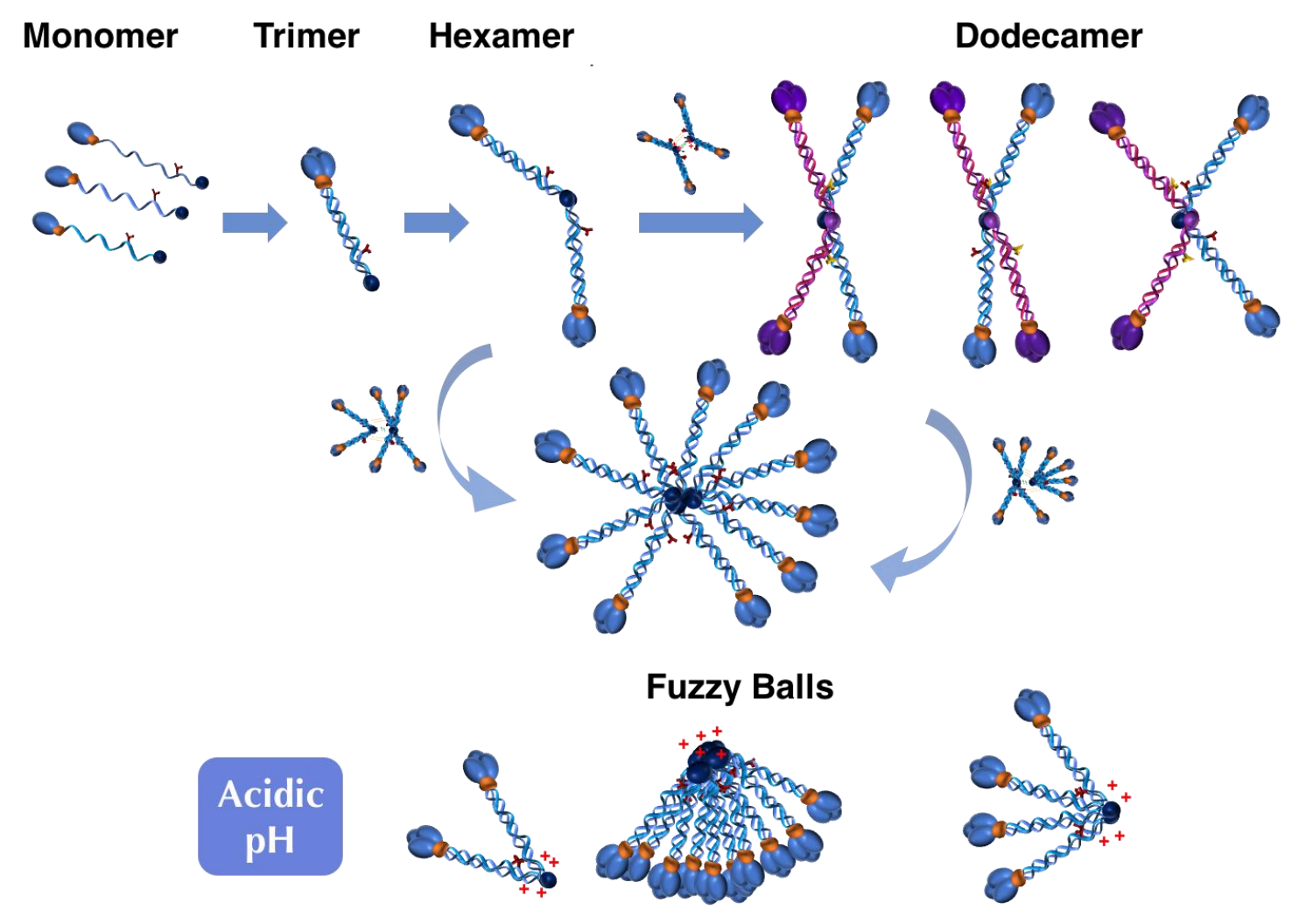


\section{SUPLEMENTARY FIGURE S1}

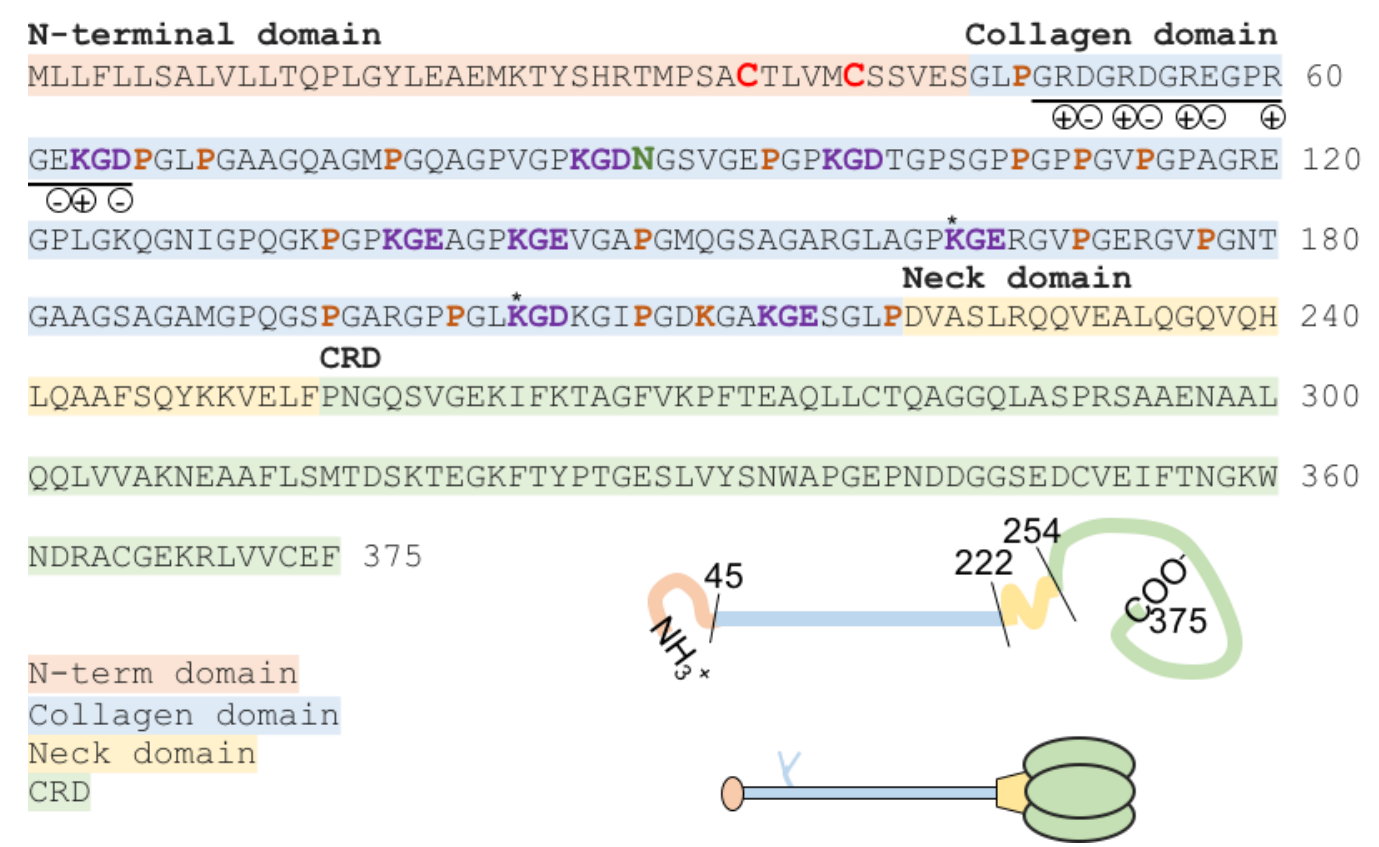

\section{Supplementary Figure S1}

\section{Amino acid sequence and domain organization of hSP-D}

The segments of the sequence defining the different protein domains are shaded with different colours: N-terminal (orange), collagen (blue), neck (yellow) and CRD (green). Cysteines $\mathrm{C}_{15}$ and $\mathrm{C}_{20} \mathrm{O}$ are in red bold letter. Glycosylated (blue $\mathrm{Y}$ at the protein scheme) residue N70 is in bold green. Hydroxylated prolines are in brown [14]. Amino acid triplets in purple represent triples prone to form ionic interactions between triple helices in the collagen region. Lysines marked with the asterisk are always found O-glycosylated, the rest of lysines within these triples can be found as partly O-glycosylated and/or hydroxylysine residues. The underlined amino acid sequence constitutes the so-called "charged patch", with the expected charge of the amino acids at pH 7 indicated below.

\section{SUPLEMENTARY FIGURE S2}


A

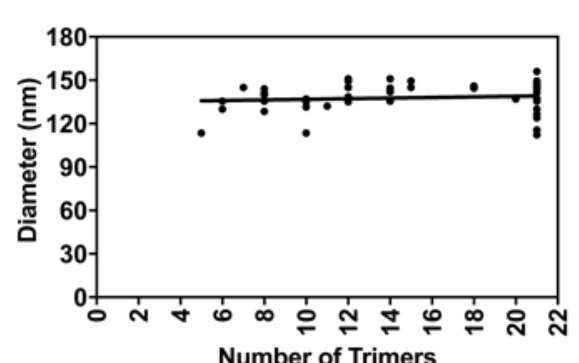

B

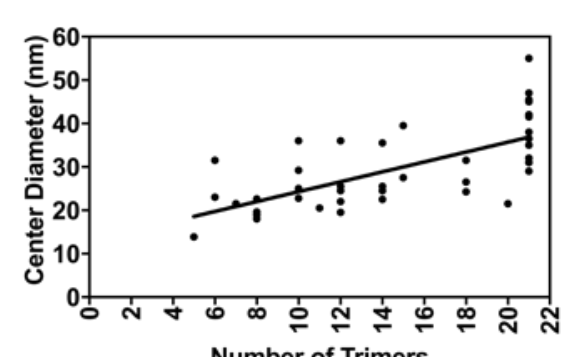

C
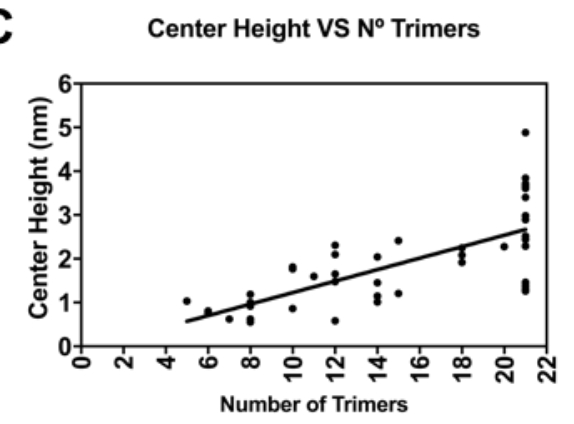
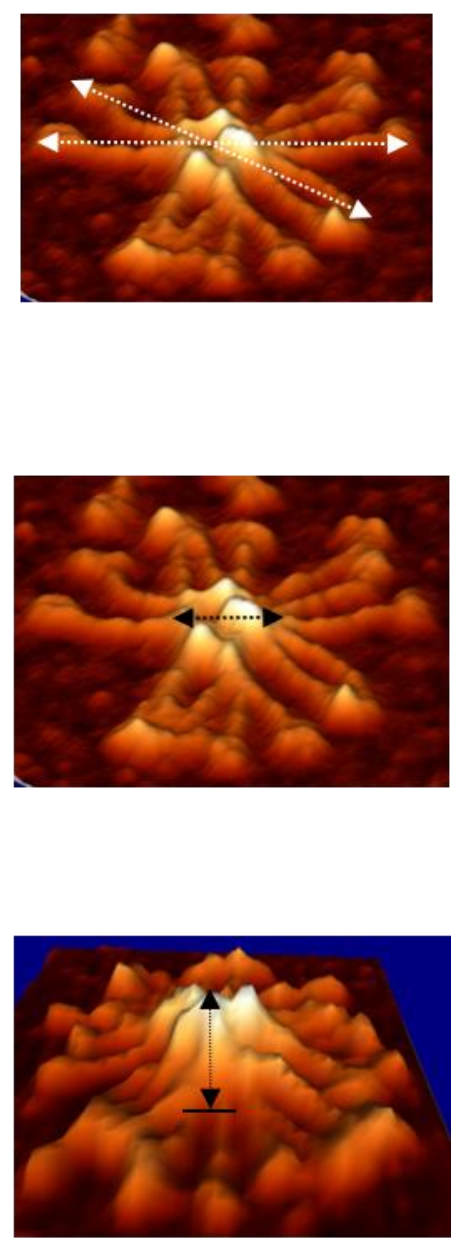

\section{Supplementary Figure S2}

\section{Structural characterization of rhSP-D fuzzy balls}

Some parameters of fuzzy balls were measured and plotted against the number of trimers per molecule. $A$, total diameter of the whole molecule, taken as the average of two diameters measured of each molecule. $B$, the center diameter, taken as the average of two measurements of the central stem of each molecule, as indicated by the black arrow. $C$, the height of the center (N-terminal domain) of each fuzzy ball, measured as indicated. 


\section{SUPLEMENTARY FIGURE S3}

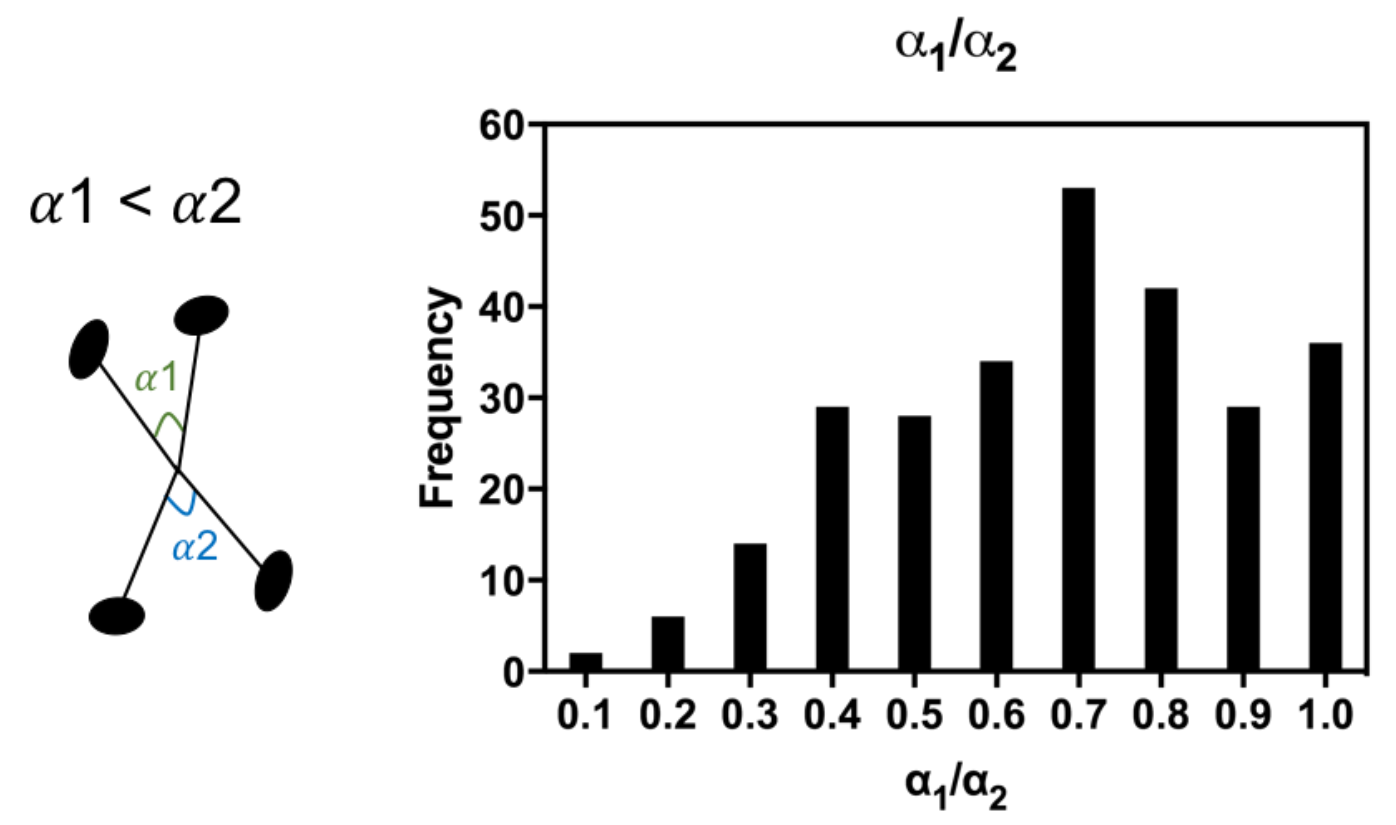

Supplementary Figure S3

Ratio of acute angles $\alpha_{1} / \alpha_{2}$ in rhSP-D dodecamers.

Frequency distribution of the ratio between the two acute angles in each of 273 dodecameric rhSP-D molecules, determined at pH 7.4. 
SUPLEMENTARY FIGURE S4
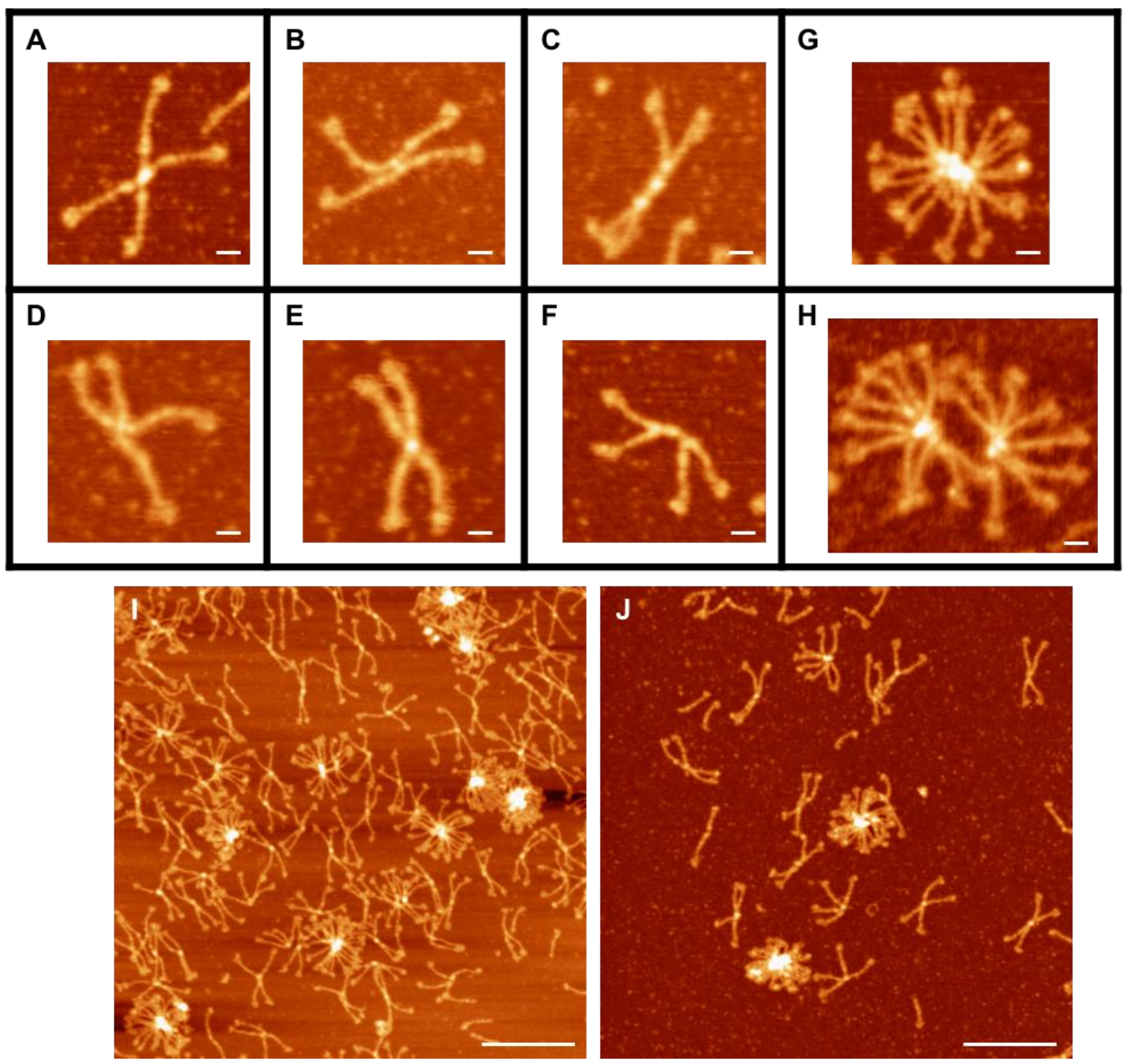

Supplementary Figure S4

AFM images of rhSP-D

$A-F$ : magnifications of some dodecamers. G-H: magnification of fuzzy balls. I-J: $1 \mu \mathrm{m}$ scan, scale bar $200 \mathrm{~nm}$. $B-H$, scale bar $20 \mathrm{~nm}$. 